\title{
HUELLA DE CARBONO- EMISIONES DE GEI POR USO DEL SISTEMA DE ILUMINACIÓN DE LA FACULTAD DE INGENIERÍA AMBIENTAL DE LA UNIVERSIDAD NACIONAL DE INGENIERÍA, LIMA-PERÚ
} CARBON FOOTPRINT- GHG EMISSIONS BY USE OF THE LIGHTING
SYSTEM OF THE FACULTY OF ENVIRONMENTAL ENGINEERING OF
THE NATIONAL UNIVERSITY OF ENGINEERING, LIMA-PERU Enrique Saavedra-Farfán ${ }^{*}$ (b)

${ }^{1}$ Facultad de Ingeniería Ambiental, Universidad Nacional de Ingeniería, Lima, Perú

Recibido (Recieved): 30/12/2019 Aceptado (Accepted): 02/03/2020

\section{RESUMEN}

El objetivo general de este estudio de investigación es contribuir a establecer parcialmente la Línea Base de la Huella de Carbono por el uso de los Sistemas de lluminación en los ambientes de las edificaciones de la Facultad de Ingeniería Ambiental (FIA) de la Universidad Nacional de Ingeniería (UNI), en el desarrollo de las actividades académicas, gestión y de apoyo. Para lo cual se establecieron los objetivos específicos: Identificar las fuentes de emisión indirecta de GEl, estimar las emisiones indirectas de GEI que provienen de la generación de electricidad de origen externo (Alcance 2.) y preparar el informe del inventario de GEI correspondientes. Las universidades y en especial las Facultades relacionadas a la Ingeniería Ambiental tienen la responsabilidad relevante de contribuir a la sostenibilidad ambiental a través de la formación académica de profesionales, con investigaciones y acciones directas al respecto; las cuales servirán académicamente para replicar las buenas prácticas en otras facultades, universidades y sectores. Esta contribución está alineada con las acciones de mitigación conformantes de las 'Contribuciones Nacionalmente Determinadas' (NDC), que constituyen el compromiso del Perú para reducir sus emisiones de gases de efecto invernadero al año 2030, en el marco del Acuerdo de París. La sociedad moderna desarrolla gran cantidad de actividades con el uso de los sistemas eléctricos, según la IEEE a nivel mundial el $66 \%$ de la electricidad es generada por combustibles fósiles y el $16 \%$ de la electricidad mundial es consumida en sistemas de iluminación; en el Perú según el Ministerio de Energía y Minas (MINEM), aproximadamente el 35\% de la energía primaria (después de la transformación y/o descontadas las pérdidas) es destinada a la generación eléctrica, de la cual el consumo energía por la iluminación en los hogares es de 19\% del total de la factura eléctrica, en el sector público este porcentaje se eleva al $20 \%$ y en el sector comercial hasta el $25 \%$. El informe del inventario de GEI se ha realizado según la Norma ISO 14064-1. La metodología de cuantificación aplicada es la combinación de medición y cálculo. Los cálculos basados en datos de las actividades de GEI, datos de las horas de consumo de energía eléctrica y las mediciones directas de consumo intermitente de energía por los sistemas de iluminación, multiplicado por el Factor de Emisión (FE) de GEl. El año base considerado es 2018, determinándose el inventario de emisiones indirectas de GEI que provienen de la generación de electricidad de origen externo (Alcance 2) por uso del Sistema de iluminación de la Facultad de Ingeniería Ambiental de la Universidad Nacional de Ingeniería, en las actividades académicas, de gestión y apoyo, de 63.169 tonCO2e. Siendo el mayor consumo por la actividad académica directa (aulas, laboratorios y biblioteca) $41.18 \%$. Una emisión per cápita de 0.061 tonCO2e/estudiante y 0.052 tonCO2e/usuario, así como un promedio de 0.042 tonCO2e/lámpara.

Palabras Clave: Huella de carbono, inventario GEI, eficacia luminosa, lámpara LED, eficiencia energética

\section{ABSTRACT}

The general objective of this research study is to contribute to partially establishing the Carbon Footprint Baseline for the use of Lighting Systems in the building environments of the Faculty of Environmental Engineering (FIA) of the National University of Engineering (UNI), in the development of academic, management and support activities. For this purpose, the following specific objectives were established: To identify the sources of indirect GHG emissions, to estimate indirect GHG emissions that come from the generation of electricity from external sources (Scope 2), and to prepare the corresponding GHG inventory report. Universities, and especially University Faculties related to Environmental Engineering, have the relevant responsibility to contribute to environmental sustainability through research, direct actions, and academic training of professionals. This will be of tremendous academic impact by replicating good practices in other faculties, universities and sectors. This contribution is aligned with the mitigation actions that are part of the Nacional Determined Contributions (NDC), which constitute Peru's commitment to reduce its greenhouse gas emissions by 2030, based upon the Paris Agreement.

\footnotetext{
* Corresponding author.:
}

E-mail: bsaavedraf@uni.pe 
Modern societies develop a large number of activities with the use of electrical systems. According to the IEEE, $66 \%$ of the electricity worldwide is generated by fossil fuels and $16 \%$ of the world's electricity is consumed in lighting systems. In Peru, according to the Ministry of Energy and Mines (MINEM), approximately 35\% of the primary energy (after the transformation and /or discounting losses) is destined for electricity generation, from which energy consumption by lighting in households accounts for $19 \%$ of the total electricity bill. In the public sector, this percentage rises to $20 \%$ and in the commercial sector up to $25 \%$. The GHG inventory report has been made according to ISO 14064-1. The methodology of applied quantification is the result of the combination of measurement and calculation. Calculations based on data on GHG activities, data on hours of electric power consumption, and direct measurements of intermittent energy consumption by lighting systems are multiplied by the GHG Emission Factor (FE). The base year considered is 2018, determining the inventory of indirect GHG emissions that come from the generation of electricity of external origin (Scope 2) by use of the Lighting System of the Faculty of Environmental Engineering of the National Engineering University, in the academic, management and support activities of 63.169 tonCO2e. The highest consumption is related to direct academic activities (classrooms, laboratories and library) with $41.18 \%$. A per capita emission of 0.061 tonCO2e / student and 0.052 tonCO2e / user, as well as an average of 0.042 tonCO2e / lamp is seen.

Keywords: Carbon footprint, GHG inventory, light efficiency, LED lamp, energy efficiency

\section{INTRODUCCIÓN}

La sostenibilidad ambiental se logrará con la suma de esfuerzos transversales a los diversos países y sectores, las universidades y en especial las facultades relacionadas a la Ingeniería Ambiental, tienen la responsabilidad relevante de contribuir al logró a través de la formación académica de profesionales, con investigaciones y acciones directas al respecto.

El objetivo general de esta investigación es contribuir a establecer parcialmente la Línea Base de la Huella de Carbono por el uso de los Sistemas de lluminación en los ambientes de las edificaciones de la Facultad de Ingeniería Ambiental (FIA) de la Universidad Nacional de Ingeniería (UNI), en el desarrollo de las actividades académicas, de gestión y apoyo. Para lo cual se establecieron los objetivos específicos: Identificar las fuentes de emisión indirecta de GEl, estimar las emisiones indirectas de GEI que provienen de la generación de electricidad de origen externo (Alcance 2) y preparar el informe del inventario de GEI correspondientes [4]; estableciendo una forma de contribuir al logro del objetivo a nivel país asumido con relación a la COP21 [1].

La sociedad moderna desarrolla gran cantidad de actividades con el uso de los sistemas eléctricos, los sectores industrial, comercial, residencial y público basan sus operaciones en su uso. A nivel mundial, el $66 \%$ de la electricidad es generada por combustibles fósiles, el 16\% por energía hidráulica, el 11\% nuclear y el $7 \%$ de energía renovable [2]. En términos de demanda, el $16 \%$ de la electricidad mundial es consumida en sistemas de iluminación [2].

En el Perú, según el Ministerio de Energía y Minas (MINEM), aproximadamente el $35 \%$ de la energía primaria (después de la transformación y/o descontadas las pérdidas) es destinada a la generación eléctrica, la cual presenta consumos importantes en iluminación: Sector Residencial $15 \%$ a $30 \%$, sector Comercial aprox. 33\%, sector Público aprox. 24\% y sector industrial $10 \%$ a $15 \%$ [3]. En cuanto a lo económico, en el Perú, el consumo energía por la iluminación en los hogares es de $19 \%$ del total de la factura eléctrica, en el sector público este porcentaje se eleva al $20 \%$ y en el sector comercial hasta el $25 \%$ [6].

Dados estos niveles de consumo en sistemas de iluminación, el uso transversal importante de la iluminación en todos los sectores, el desafío en la demanda es que los sistemas utilicen la energía eléctrica de manera eficiente, en nuestro caso los sistemas de iluminación, para que nuestros sistemas energético, ambiental y económico sean sostenibles [2].

El informe ha sido desarrollado de acuerdo con los principios y requerimientos para la presentación de informes sobre los inventarios de Gases Efecto Invernadero establecidos en la Norma ISO 14064-1 [7].

En los siguientes capítulos se presenta la justificación e importancia del estudio, el marco teórico respecto a la Huella de Carbono, descripción de la norma ISO 14064-1, el informe correspondiente del inventario de GEI, oportunidades de mejora tecnológicas y de gestión de uso y las conclusiones del estudio.

Este proyecto de investigación es parte complementaria del desarrollo de la tesis doctoral del Jefe de Proyecto, la cual está relacionada con la eficiencia energética de la demanda y comprende aspectos energéticos, ambientales y económicos.

\subsection{JUSTIFICACIÓN E IMPORTANCIA}

Después del Acuerdo de París (COP21-2015), los países han iniciado el camino hacia una economía baja en emisiones de carbono. Se ha fijado un tope a las emisiones mundiales para mantener el aumento de la temperatura media mundial muy por debajo de 2 grados Celsius en el largo plazo. Los compromisos climáticos de todos los países ante la Organización de las Naciones Unidas (ONU) implican una transición 
significativa del sector eléctrico y la eficiencia energética en los próximos quince años. El compromiso climático del Perú contempla una reducción del $30 \%$ respecto a las emisiones de gases de efecto invernadero (GEI) proyectadas para el año 2030, como parte de un escenario sin cambios o business as usual (BaU). Al año 2030, si el Perú no realizará ningún esfuerzo para controlar el crecimiento de sus emisiones de gases de efecto invernadero, estas casi se duplicarían respecto del 2010. Esto significaría mantener altos niveles de deforestación, bajos niveles de productividad y eficiencia en el uso de la energía. [8]

La falta de un estudio de caracterización energética en la Facultad de Ingeniería Ambiental, así como la falta de información sobre los consumos de energía útil en iluminación, son factores que determinaron la concepción general de los objetivos del Proyecto. Los estudios realizados y la experiencia obtenida en eficiencia energética se han centralizado en las grandes empresas. Por ello, obtener información sobre el consumo de energía útil en fuentes bibliográficas es una tarea ardua, tratando de obtener indicadores, características, capacitación, proyectos ya realizados, entre otros. En este sentido, dada la importante pero limitada fuente de información se encuentra información sobre experiencias en otros países, pero se necesita adaptar esta información a las características del país y que nuestra Facultad de Ingeniería Ambiental cuente con su propia información de la Huella de Carbono y la contribución de este estudio es por el uso de los Sistemas de Iluminación en los ambientes de las edificaciones de la FIA-UNI. [5]

Por lo que la Facultad de Ingeniería Ambiental de la Universidad Nacional de Ingeniería, como actividad inicial debe tener conocimiento de su correspondiente Huella de Carbono y de esta forma poder establecer metas y estrategias adecuadas para su reducción y mitigación de impactos. Los cuales servirán académicamente para replicar las buenas prácticas en otras Facultades, Universidades y sectores.

Los aportes de este proyecto de investigación permitirán desarrollar actividades de sensibilización y difusión de información y de los resultados, dando a conocer las ventajas y beneficios de la gestión de la emisión de GEI al sector público, sector empresarial y sociedad civil, mediante la eficiencia energética en el uso de los sistemas de iluminación. Lo que se busca finalmente es contribuir a un cambio cultural en la sociedad, mediante el cual los conceptos de ambiente sostenible, eficiencia energética y uso de energías limpias estén incorporados y vinculados en las actividades cotidianas [5].

\section{HUELLA DE CARBONO}

La Huella de Carbono es una métrica ambiental que permite cuantificar de forma objetiva las emisiones de Gases Efecto Invernadero (GEI) que se generan de manera directa e indirecta por la realización de diversas actividades realizadas por organizaciones públicas 0 privadas, al producir un producto o brindar un servicio, o por el desarrollo de un proyecto o evento. Se obtiene información fundamental para la gestión a fin de mitigar y/o compensar las emisiones e identificar oportunidades de mejora que impactan en la contribución del logro de los objetivos comprometidos respecto al calentamiento global y el cambio climático, así como la satisfacción de clientes, rentabilidad y valor de la marca, a nivel institucional.

La Huella de Carbono se define como la cantidad total de GEI que se generan de manera directa e indirecta por la realización de diversas actividades realizadas por organizaciones públicas o privadas, al producir un producto o brindar un servicio, o por el desarrollo de un proyecto o evento [9]. Es por tanto un inventario de $\mathrm{GEI}$, que se mide en toneladas de $\mathrm{CO}_{2}$ equivalentes y que tiene en cuenta los seis tipos de gases considerados en el Protocolo de Kioto $\left(\mathrm{CO}_{2}, \mathrm{CH}_{4}\right.$, N2O, PFCs, HFCs y SF6) [10], [11].

La huella de carbono de organizaciones, también llamada inventario de GEI corporativo, mide las emisiones de GEI derivadas de todas las actividades de una organización [10]; a nivel de proyecto, cuantifica la reducción de emisiones o el aumento en las remociones de gases de efecto invernadero [12]; en el caso de un producto o servicio, el inventario comprende las emisiones a lo largo de todo el ciclo de vida del producto o servicio, desde la obtención de la materia prima hasta que se convierte en residuo (enfoque desde la cuna a la tumba) o se recicla (desde la cuna a la cuna), aunque también se pueden utilizar enfoques que finalizan en la siguiente organización que utilizará el producto como su materia prima (desde la cuna a la puerta) [10].

El exceso de emisión de los GEI producto de la actividad humana y carbonización de la producción, contribuye directamente al calentamiento global, lo cual impacta en el cambio climático, según los informes del IPCC (Grupo Intergubernamental sobre el Cambio Climático) [13].

Desde la entrada del Protocolo de Kioto, cada vez son más las organizaciones que tienen la necesidad de reducir o controlar sus emisiones. Esto tiene como consecuencia los compromisos suscritos tanto por los sectores de diversos países, dada la globalización de los mercados, de organizaciones por obligación 
reglamentaria como voluntariamente por las indirectamente vinculadas [11].

Motivaciones internas: definir objetivos y políticas de reducción efectiva de GEl, identificando oportunidades de reducciones de energía y ahorros de costos asociados [11]. Motivaciones externas: principalmente elevar la reputación de marca, evidenciando los compromisos de responsabilidad social y ambiental [11].

La gestión de la huella de carbono comprende conocer el nivel de emisiones, identificar opciones de mejora, desarrollar planes de acción con posibles acciones para reducir y/o compensar (carbono neutro) la emisión de GEI [11].

Existen normas ISO que facilitan el cálculo y gestión de la huella de carbono; se espera que beneficie a las organizaciones, los gobiernos, los proponentes de proyectos y las partes interesadas; proporcionando claridad y coherencia para la cuantificación, el seguimiento, el informe y la validación o verificación de los proyectos e inventarios de GEI para facilitar el desarrollo de estrategias y planes de gestión de GEI [14].

\section{- Para organizaciones: ISO 14064-1:2018}

Especifica los principios y requisitos a nivel de la organización para la cuantificación y notificación de emisiones y absorciones de gases de efecto invernadero (GEI). Incluye requisitos para el diseño, desarrollo, gestión, informes y verificación del inventario de GEI de una organización [15].

\section{- Para proyectos: ISO 14064-2:2019}

Especifica los principios y requisitos y proporciona orientación a nivel de proyecto para la cuantificación, el monitoreo y la presentación de informes de actividades destinadas a causar reducciones o remociones de emisiones de gases de efecto invernadero (GEI). Incluye requisitos para planificar un proyecto de GEI, identificar y seleccionar fuentes, sumideros y depósitos de GEI relevantes para el proyecto y el escenario de referencia, monitorear, cuantificar, documentar e informar el desempeño del proyecto de GEl y administrar la calidad de los datos [16].

\section{- Para productos: ISO 14067:2018}

Este documento especifica los principios, requisitos y pautas para la cuantificación y la notificación de la Huella de Carbono de un Producto (HCP), de manera consistente con las Normas Internacionales de evaluación del ciclo de vida (ISO 14040 e ISO 14044).
También se especifican los requisitos y las pautas para la cuantificación de una HCP parcial [17].

\section{- Para verificación: ISO 14064-3:2019}

-

Este documento especifica los principios y requisitos y proporciona orientación para verificar y validar las declaraciones de gases de efecto invernadero (GEI).

Es aplicable a las declaraciones de GEI de organización, proyecto y producto [18].

\section{ISO 14064-1 [14]}

Proporciona claridad y coherencia para la cuantificación, el seguimiento, el informe y la validación o verificación de los inventarios de GEI de la organización, para facilitar el desarrollo de estrategias y planes de gestión de GEI [14].

Debido a que el estudio corresponde a las actividades realizadas al interior de la organización (FIA), se aplicó la norma ISO 14064-1, por lo que en este capítulo describiremos los aspectos generales comprendidos por esta norma y la aplicación específica se desarrolla y presenta en el informe, en el Capítulo 4.

La norma comprende:

\subsection{OBJETO Y CAMPO DE APLICACIÓN}

Esta norma tiene por objeto especificar los principios y requisitos para la cuantificación y el informe de emisiones y remociones de GEl y el campo de aplicación es a nivel de la organización. Incluye los requisitos para diseño, desarrollo, gestión, informe y/o verificación de un inventario de GEI de una organización [7].

\subsection{TÉRMINOS Y DEFINICIONES}

Comprende los términos y definiciones, aplicados en la norma [7].

\subsection{PRINCIPIOS [7]}

Para asegurarse que la información relacionada con los GEI es cierta e imparcial. Son la base para los requisitos y guían su aplicación:

- Pertinencia: Seleccionar las fuentes, sumideros y reservorios, datos y metodologías apropiados para las necesidades del usuario.

- Cobertura total: Incluir todas las emisiones y remociones pertinentes de GEl.

- Coherencia: Permitir comparaciones significativas en la información relacionada con los GEI.

- Exactitud: Reducir el sesgo y la incertidumbre, posible. 
- Transparencia: Divulgar información suficiente y apropiada, que permita tomar decisiones con confianza razonable.

\subsection{DISEÑO Y DESARROLLO DEL INVENTARIO DE GEI [7]}

Límites de la organización: La organización, puede estar compuesta de varias instalaciones y cada una puede tener varias fuentes o sumideros de GEI; debe consolidar sus emisiones y remociones de GEI a nivel de instalación según uno de los dos siguientes enfoques:

- Control: Considera las emisiones y remociones de GEI sobre las instalaciones que tiene:

- Control operacional: Responde por las instalaciones que opera.

- Control financiero: Responde por las instalaciones que financia.

- Cuota de participación correspondiente: Considera las emisiones y remociones de GEI de su parte de las respectivas instalaciones.

La organización puede utilizar diferentes metodologías de consolidación y cuando la instalación es controlada por varias organizaciones, estas deben aplicar la misma metodología. El método debe documentarse y debe explicar cualquier cambio al método aplicado.

- Límites operativos: La organización debe identificar las emisiones y remociones de GEI asociadas a las operaciones, clasificándolas en:

- Emisiones y remociones directas de GEI (Alcance 1): Cuantifica las emisiones provenientes de las instalaciones y las remociones de las instalaciones, dentro de los límites de la organización. Se puede informar por separado las emisiones provenientes de la electricidad, calor y el vapor generado y exportado o distribuido por la organización, pero no se deben deducir de las emisiones directas totales de la organización.

- Emisiones indirectas de GEI por energía (Alcance 2): Cuantifica las emisiones indirectas que provienen de la generación de electricidad, calor o vapor de origen externo, consumido por la organización.

- Otras emisiones indirectas de GEI (Alcance 3): Cuantifica emisiones indirectas diferentes a las ya consideradas en el Alcance 2.

- Cuantificación de emisiones y remociones de GEI

Se debe cuantificar y documentar las emisiones y remociones, completando según sea aplicable las etapas y exclusiones siguientes:

- Identificación de fuentes y sumideros de GEI: Que contribuyen a las emisiones directas, se debería documentar por separado los proveedores de electricidad, calor o vapor importados consumidos por la organización.
- Selección de metodologías de cuantificación: Seleccionar y usar metodologías de cuantificación que minimicen razonablemente la incertidumbre y produzcan resultados exactos, coherentes y reproducibles. Los cuales se pueden clasificar en los siguientes tipos:

Cálculos basados en: datos de la actividad de GEI por los factores de emisión o remoción; el uso de modelos; correlaciones específicas para la instalación; enfoque relacionado con los balances de masa. Medición continua o intermitente. O la combinación de medición y cálculo.

- Selección y recopilación de datos de la actividad: Si se emplean, deben ser coherentes con la metodología seleccionada.

- Selección y/o desarrollo de factores de emisión o remoción de GEI: Si se emplean deben derivarse de un origen reconocido; sean apropiados para las fuentes y sumideros, involucrados; estén actualizados; que la incertidumbre y los cálculos produzcan resultados exactos y reproducibles y sean coherentes con el uso previsto del inventario.

- Cálculo de emisiones y remociones: De acuerdo con la metodología de cuantificación seleccionada.

La organización puede excluir de la cuantificación las fuentes o sumideros de GEI directas o indirectas cuya contribución no es importante o cuya cuantificación no sería técnicamente viable ni rentable, explicando porque se excluyen. Así mismo debe explicar la selección de la metodología seleccionada.

\subsection{COMPONENTES DEL INVENTARIO GEI [7]}

- Emisiones y remociones: Documentar por separado en las instalaciones y en la organización las emisiones directas de cada GEI, remociones, emisiones indirectas por energía, otras emisiones indirectas, emisiones directas de $\mathrm{CO}_{2}$ a partir de la combustión de biomasa. Se debe utilizar toneladas como la unidad de medida y convertir cada tipo de GEI a toneladas de $\mathrm{CO}_{2}$ usando los apropiados Potenciales de Calentamiento Global (PCG) de los Gases de Efecto Invernadero (GEI), incluidos en el Anexo $C$ de la norma [7].

- Actividades de la organización para reducir las emisiones de GEI o incrementar remociones: La organización puede planificar e implementar acciones dirigidas cuantificadas según la Norma ISO 14064-1, o proyectos de reducción de emisiones o de aumento de remociones, cuantificadas siguiendo las especificaciones de la Norma ISO 14064-2.

- Inventario de GEI del año base: Histórico, usando datos representativos de la actividad de la organización, habitualmente de un solo año, un promedio de varios años, o un promedio móvil. Con propósitos de comparación, de cumplimiento de 
objetivos u otros usos previstos. La organización debe desarrollar, aplicar y documentar un procedimiento para recalcular su año base, el cual no debe ser por cambios en los niveles de producción.

- Evaluación y reducción de la incertidumbre: Para emisiones y remociones incluyendo la asociada con los factores de emisión y remoción.

\subsection{GESTIÓN DE LA CALIDAD DEL INVENTARIO DE GEI}

- Gestión de la información: La organización debe establecer y mantener procedimientos de gestión de la información sobre los GEI, que aseguren el cumplimiento de los principios de la Norma ISO 14064. Estos procedimientos deberían considerar la identificación y revisión de la responsabilidad y autoridad, formación del equipo, límites de la organización, fuentes y sumideros, metodologías, equipo de medición, recopilación de datos, exactitud, auditorías internas, así como la revisión periódica de las oportunidades para mejorar los procesos de gestión de la información [7].

- Retención de documentos y mantenimiento de registros: Establecer y mantener procedimientos de retención de documentos y mantenimiento de registros que respalda el diseño, desarrollo y mantenimiento del inventario, que permita su verificación [7].

\subsection{INFORMES SOBRE GEI}

En el Capítulo 4. INFORME, se presenta el detalle y la aplicación de estás especificaciones. Que comprende [7]:

- Planificación del informe sobre GEl.

- Contenido del informe de la organización sobre GEI:

- El inventario de GEI que debe describir la organización.

- La información sobre GEI que debería considerarse incluir en el informe.

\subsection{FUNCIONES DE LA ORGANIZACIÓN EN LAS ACTIVIDADES DE VERIFICACIÓN}

Para la revisión objetiva e imparcial de la declaración de las emisiones o remociones de GEI frente a los requisitos de la Norma ISO 14064-3. Las cuales comprenden [7]:

- Preparación para la verificación

- Gestión de la verificación

- Plan de verificación para la organización

- Proceso de verificación

- Competencia de los verificadores

- Declaración de verificación

\section{INFORME [10] \\ 4.1 Contenido del informe sobre GEI}

Para nuestro estudio se aplica la norma ISO 14064-1, debido a que comprende la cuantificación e informe de los inventarios de GEI correspondientes parcialmente a las actividades de la organización, específicamente por el uso de los sistemas de iluminación de la Facultad de Ingeniería Ambiental de la Universidad Nacional de Ingeniería.

El informe de la organización sobre GEI describe el inventario e incluye:

\section{a. Descripción de la organización informante}

Este informe corresponde al aporte para determinar parcialmente la Línea Base de la Huella de Carbono por otras emisiones indirectas de GEI que provienen de la generación de electricidad de origen externo (Alcance 2.) consumida por el Sistema de lluminación en el desarrollo de las actividades académicas, de gestión y apoyo al interior de los ambientes de la Facultad de Ingeniería Ambiental (FIA), de la Universidad Nacional de Ingeniería (UNI), cuya sede está ubicada en el campo universitario en la Av. Túpac Amaru s/n, distrito de Rímac, en la ciudad de Lima, Perú.

El estudio, cuantificación e informe ha sido desarrollado por el equipo de investigación, liderado por el autor de este artículo, de un proyecto de investigación formativa, promovido por la Universidad Nacional de Ingeniería (UNI), el Vicerrectorado de Investigación (VRI) y la Facultad de Ingeniería Ambiental (FIA), en cumplimiento de la función promotora, para el desarrollo científico y tecnológico; cuyo proceso, en la facultad, es delegado a la Unidad de Investigación de la FIA.

La Universidad Nacional de Ingeniería (UNI), según los art. $1^{\circ}$, art. $2^{\circ}$ y art. $36^{\circ}$ de su Estatuto aprobado el 14 de diciembre del 2014, en el marco de la Ley Universitaria $\mathrm{N}^{\circ}$ 30220-2014, se constituye como 'una persona jurídica de derecho público interno, con sede en la ciudad de Lima. Se rige por su estatuto y reglamentos en el marco de la Constitución y las leyes' y se describe como 'una comunidad académica, orientada a la investigación y a la docencia, que brinda una formación humanista, científica y tecnológica con una clara conciencia de nuestro país como realidad multicultural. Adopta el concepto de educación como derecho fundamental y servicio público esencial y está integrada por docentes, estudiantes y graduados' [19], [20], [21]. Se estructura 'por Facultades que son unidades básicas de organización. Las Facultades están compuestas por Departamentos Académicos, Escuelas Profesionales, Unidad(es) o Instituto(s) de Investigación, Unidad de Posgrado, Laboratorios y Unidades de Servicio' [20]. 


\section{b. Persona responsable}

El jefe del proyecto 'Huella de carbono - Emisiones de GEI por uso del Sistema de iluminación de la Facultad de Ingeniería Ambiental de la Universidad Nacional de Ingeniería, Lima-Perú' y autor de este artículo, es el responsable del informe. Cuya metodología de cuantificación aplicada, corresponde a cálculos basados en datos de la actividad de GEI multiplicados por el factor de emisión, como parte del desarrollo de un proyecto de investigación formativa, patrocinado por la Facultad de Ingeniería Ambiental a través de la Unidad de Investigación. Así mismo, los resultados son conformantes parciales de la tesis para obtener el grado de doctor en ciencias con mención en energética, en desarrollo también por el autor de este artículo.

\section{c. Periodo que cubre el informe}

El siguiente informe reporta las emisiones indirectas de GEI que provienen de la generación de electricidad de origen externo, para las 52 semanas del año 2018, por el uso del sistema de iluminación para realizar las actividades académicas, de gestión y de apoyo, identificadas en la Facultad de Ingeniería Ambiental, detalladas en la tabla I.

El año académico 2018, está compuesto por los dos períodos académicos: 2018-1 (de semana 1 a 30) y el 2018-2 (de la semana 31 a 52), de acuerdo con los cursos se desarrolla la carga horaria para el ciclo académico 2018-1 (semanas 11 a 17 y 19 a 25) y para el ciclo académico 2018-2 (semanas 34 a 40 y 42 a 48), la carga horaria comprende la programación de horarios de uso de aulas y laboratorios, se considera el uso durante las semanas de clases como $100 \%$ de lo programado y el porcentaje de uso durante el resto de semanas y ambientes se indica en la tabla I. La ocupación de los ambientes durante las 52 semanas del año se diferencia en 2 tipos de uso semanal (ver TABLA I):

- Semana tipo 1: 14 semanas durante las clases del ciclo 2018-1 y 14 semana durante clases del ciclo 2018-2 (aulas, laboratorios y otros ambientes al 100\% de lo programado).

- Semana tipo 2: 24 semanas restantes (durante las cuales las aulas y laboratorios, se usan al 50\% de lo programado para la semana tipo 1 y los otros ambientes siempre al 100\%).

El detalle del porcentaje de ocupación de todos los ambientes se muestra en la tabla I.

$$
\text { TABLA I }
$$

Actividad desarrollada en la FIA / semana y \% de uso - 2018

\begin{tabular}{|c|c|c|c|c|}
\hline \multirow[t]{2}{*}{ Semana } & \multirow[t]{2}{*}{ Actividad } & \multicolumn{3}{|c|}{ Ocupación } \\
\hline & & Aulas & Laboratorios & Otros \\
\hline 01 a 06 & $\begin{array}{c}\text { Actividades } \\
\text { preparatorias 2018-1 }\end{array}$ & $50 \%$ & $50 \%$ & $100 \%$ \\
\hline 07 & Admisión 2018-1 & $50 \%$ & $50 \%$ & $100 \%$ \\
\hline 08 a 10 & $\begin{array}{c}\text { Actividades } \\
\text { preparatorias 20181-1 }\end{array}$ & $50 \%$ & $50 \%$ & $100 \%$ \\
\hline 11 a 17 & Clases 2018-1 & $100 \%$ & $100 \%$ & $100 \%$ \\
\hline
\end{tabular}

\begin{tabular}{|c|c|c|c|c|}
\hline 18 & $\begin{array}{l}\text { Exámenes parciales } \\
\qquad 2018-1\end{array}$ & $50 \%$ & $50 \%$ & $100 \%$ \\
\hline 19 a 25 & Clases 2018-1 & $100 \%$ & $100 \%$ & $100 \%$ \\
\hline 26 & Exámenes finales 2018-1 & $50 \%$ & $50 \%$ & $100 \%$ \\
\hline 27 y 28 & $\begin{array}{l}\text { Exámenes sustitutorios } \\
\qquad 2018-1\end{array}$ & $50 \%$ & $50 \%$ & $100 \%$ \\
\hline 29 y 30 & Cierre académico 2018-1 & $50 \%$ & $50 \%$ & $100 \%$ \\
\hline 31 & $\begin{array}{l}\text { Actividades } \\
\text { preparatorias 2018-2 }\end{array}$ & $50 \%$ & $50 \%$ & $100 \%$ \\
\hline 32 & Admisión 2018-2 & $50 \%$ & $50 \%$ & $100 \%$ \\
\hline 33 & $\begin{array}{l}\text { Actividades } \\
\text { preparatorias 2018-2 }\end{array}$ & $50 \%$ & $50 \%$ & $100 \%$ \\
\hline 34 a 40 & Clases 2018-2 & $100 \%$ & $100 \%$ & $100 \%$ \\
\hline 41 & $\begin{array}{l}\text { Exámenes parciales } \\
\qquad 2018-2\end{array}$ & $50 \%$ & $50 \%$ & $100 \%$ \\
\hline 42 a 48 & Clases 2018-2 & $100 \%$ & $100 \%$ & $100 \%$ \\
\hline 49 & Exámenes finales 2018-2 & $50 \%$ & $50 \%$ & $100 \%$ \\
\hline 50 y 51 & $\begin{array}{l}\text { Exámenes sustitutorios } \\
\qquad 2018-2\end{array}$ & $50 \%$ & $50 \%$ & $100 \%$ \\
\hline 52 & Cierre académico 2018-2 & $50 \%$ & $50 \%$ & $100 \%$ \\
\hline
\end{tabular}

Nota: Elaboración propia

\section{d. Documentación de los límites de la organización (véase [7] numeral 4.1)}

La Universidad (UNI) comprende 11 facultades, de las cuales la Facultad de Ingeniería Ambiental (FIA) para el propósito de este estudio es considerada la organización, debido a que el inventario corresponde a la emisión indirecta $\mathrm{GEI}$ que provienen de la generación de electricidad de origen externo por el uso del sistema de iluminación al interior de sus instalaciones. No se ha considerado fuentes directas, sumideros ni otras emisiones indirectas por estar fuera del objetivo del proyecto. Se ha consolidado las emisiones indirectas de GEI a nivel de instalación según el enfoque de Control Operacional y responde por las emisiones indirectas de GEI de las instalaciones que controla y opera la facultad (FIA).

Para el propósito del inventario de GEl, las instalaciones de la Facultad de Ingeniería Ambiental han sido estructuradas en 5 edificios concurrentes al interior del área de la FIA, numerados del 1 al 5, en los cuales se desarrollan las siguientes actividades (ver TABLA II):

TABLA II

Actividad desarrollada/edificio

\begin{tabular}{lccccc}
\hline Ambientes & Edif.1 & Edif.2 & Edif.3 & Edif.4 & Edif.5 \\
\hline Académicas & $\mathrm{Si}$ & $\mathrm{Si}$ & $\mathrm{Si}$ & $\mathrm{Si}$ & $\mathrm{Si}$ \\
Gestión & $\mathrm{Si}$ & $\mathrm{No}$ & $\mathrm{No}$ & $\mathrm{Si}$ & $\mathrm{Si}$ \\
Apoyo & $\mathrm{Si}$ & $\mathrm{Si}$ & $\mathrm{No}$ & $\mathrm{Si}$ & $\mathrm{Si}$ \\
\hline
\end{tabular}

Nota: Elaboración propia

- Edificio 1 (FIA D2): Pabellón principal, comprende las oficinas del Decanato y de las Direcciones de las Escuelas Profesionales, donde se desarrollan actividades académicas y de gestión; aulas, auditorios y oficinas de los docentes, donde se desarrollan actividades académicas de enseñanza-aprendizaje; sala de reuniones donde se desarrollan actividades académicas y de gestión; y oficinas donde se desarrollan actividades de apoyo. 
- Edificio 2 (FIA D4): Centro de Tecnologías de Comunicación (CETEC), comprende los laboratorios de enseñanza-aprendizaje de Tecnologías de la Información y Comunicaciones (TIC); oficinas de apoyo donde se desarrollan actividades de tutoría de estudiantes, tecnológicas y administrativas; y aulas donde se desarrollan actividades de enseñanzaaprendizaje.

- Edificio 3 (FIA D4): Aulas, donde se desarrollan actividades de enseñanza-aprendizaje.

- Edificio 4 (FIA D4): Laboratorios y oficinas, donde se desarrollan actividades de enseñanza-aprendizaje e investigación, así como actividades de gestión y apoyo.

- Edificio 5 (FIA D2): Unidad de Posgrado, Unidad de Investigación y bibliotecas, donde se desarrollan actividades académicas, gestión y apoyo; así como de apoyo al aprendizaje e investigación.

Los 5 edificios comprenden también áreas comunes: patios, pasadizos, escaleras, baños, así como cafeterías de uso común. Los límites de la organización se grafican en la Fig. 1.

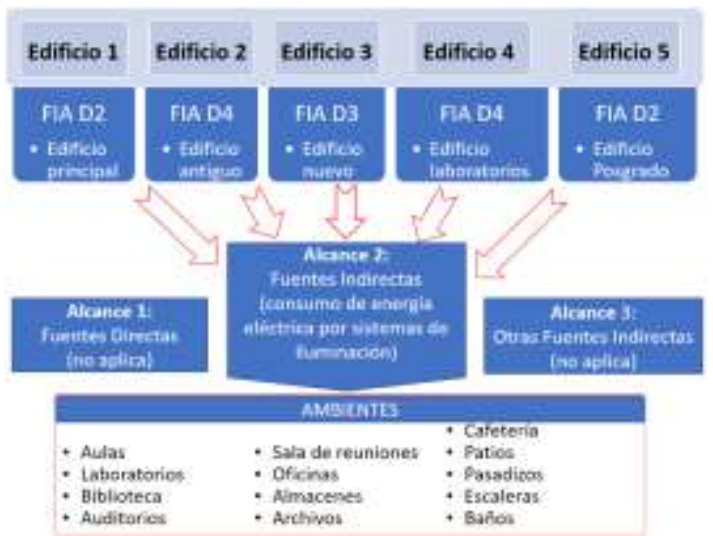

Fig. 1. Límites de la organización - Facultad de Ingeniería Ambiental.

e. Emisiones directas de GEI, cuantificadas por separado para cada GEI, en toneladas de $\mathrm{CO}_{2} \mathrm{e}$ (véase [7] numeral 4.2.2)

Las emisiones directas no corresponden al objetivo de este proyecto.

f. Una descripción de cómo se considerarán en el inventario de GEI las emisiones de $\mathrm{CO}_{2}$, a partir de la combustión de la biomasa (véase [7] numeral 4.2.2)

Las emisiones de la combustión de la biomasa no corresponden al objetivo de este proyecto.

g. Si se cuantifican las remociones de GEI hacerlo
en toneladas de CO2e (véase [7] numeral 4.2.2)

Las remociones no corresponden al objetivo de este proyecto.

\section{h. Explicar las razones para la exclusión de la cuantificación de cualquier fuente o sumidero de GEI (véase [7] numeral 4.3.1)}

Dada la importante pero limitada información, los niveles relevantes de consumo de energía eléctrica por los sistemas de iluminación (presentados en el capítulo 1) y el uso transversal en todos los ambientes, se necesita que la Facultad de Ingeniería Ambiental cuente con su propia información de la Huella de Carbono y el objetivo de este estudio es contribuir parcialmente con el inventario de emisiones indirectas GEI que provienen de la generación de electricidad de origen externo, por el uso de los Sistemas de Iluminación en los ambientes de las edificaciones de la FIA_UNI [5], para realizar las actividades académicas, gestión y apoyo (Alcance 2) [3].

El objetivo de este proyecto no comprende el inventario de GEI de fuentes de emisiones directas ni sumideros (Alcance 1), ya que se ha limitado específicamente a emisiones indirectas GEI que provienen de la generación de electricidad de origen externo por el consumo del sistema de iluminación, para realizar las actividades académicas, gestión y apoyo (Alcance 2) identificadas en la FIA, por lo que tampoco comprende otras emisiones indirectas (Alcance 3).

i. Emisiones indirectas de GEI por energía asociada con la generación de electricidad, calor o vapor de una fuente externa, cuantificadas por separado en toneladas de CO2e (véase [7] numeral 4.2.3)

De acuerdo con el objetivo de este estudio, se cuantifica las emisiones indirectas que provienen de la generación de electricidad de origen externo (Alcance 2), consumida por los sistemas de iluminación en los ambientes de las instalaciones de la FIA, que comprende 5 edificios (ver TABLA III) donde se desarrollan las actividades académicas, gestión y de apoyo, presentadas en la tabla II:

TABLA III

Cantidad de ambientes/edificio

\begin{tabular}{lrrrrrr}
\hline Ambientes & Edif.1 & Edif.2 & Edif.3 & Edif.4 & Edif.5 & Total \\
\hline Aula & 10 & 4 & 6 & 0 & 2 & 22 \\
Laboratorio & 0 & 4 & 0 & 16 & 0 & 20 \\
Biblioteca & 0 & 0 & 0 & 0 & 2 & 2 \\
Auditorio & 2 & 0 & 0 & 0 & 0 & 2 \\
Sala de reuni & 1 & 0 & 0 & 0 & 0 & 1 \\
Oficina & 23 & 2 & 0 & 3 & 5 & 33 \\
Almacén & 3 & 0 & 2 & 0 & 0 & 3 \\
Archivo & 1 & 0 & 0 & 0 & 2 & 3 \\
Cafetería & 2 & 0 & 1 & 0 & 0 & 3 \\
Patio & 9 & 5 & 2 & 1 & 0 & 17 \\
Pasadizo & 0 & 3 & 3 & 0 & 1 & 7 \\
Escalera & 8 & 3 & 6 & 0 & 2 & 19 \\
Baño & 16 & 2 & 3 & 0 & 4 & 25 \\
\hline
\end{tabular}

Nota: Elaboración propia 
Los 5 edificios comprenden ambientes donde se tienen instalados sistemas de iluminación de los tipos presentados en la tabla IV, cuyas características fotométricas se detallan en la tabla $V$ y en la tabla VI las características eléctricas nominales y los valores medidos directamente.

TABLA IV

Cantidad de lámparas/edificio

\begin{tabular}{lcccccc}
\hline Lámparas & Edif.1 & Edif.2 & Edif.3 & Edif.4 & Edif.5 & Total \\
\hline LED 1700 & 89 & 20 & 0 & 0 & 0 & 109 \\
LED 1600 & 221 & 172 & 12 & 0 & 0 & 405 \\
LED panel & 27 & 0 & 0 & 0 & 0 & 27 \\
cuadrado & & & & & & \\
LED panel & 1 & 0 & 0 & 0 & 0 & 1 \\
rectangular & & & & & & \\
LED panel & 9 & 0 & 3 & 0 & 0 & 12 \\
circular & 11 & 0 & 0 & 0 & 0 & 11 \\
LED dicroica & 2 & 1 & 0 & 0 & 0 & 3 \\
LED reflector & 2 & 0 & 0 & 4 & 115 & 219 \\
T12m & 100 & 2 & 1 & 0 & 86 & 173 \\
T8m & 84 & 16 & 28 & 42 & 0 & 118 \\
T8e & 32 & 0 & 0 & 0 & 0 & 1 \\
T8m60 & 1 & 0 & 0 & 0 & 0 & 6 \\
T8e60 & 6 & 0 & 178 & 112 & 0 & 314 \\
T5e120 & 24 & 0 & 0 & 0 & 0 & 88 \\
T5e060 & 88 & 0 & 0 & 0 & 4 & 5 \\
Circular & 0 & 1 & 0 & 0 & 0 & 13 \\
LFC & 6 & 6 & 1 & 0 & 0 & 2 \\
SAP 150W & 1 & 1 & 0 & 0 & 0 & 2 \\
MAP 250W & 2 & 0 & 0 & & & 1509 \\
\hline & & & & & & \\
\hline Nota: Elaboracion & propia & & & & & \\
\hline
\end{tabular}

Nota: Elaboración propia

La cantidad de horas de consumo de energía por los sistemas de iluminación en cada ambiente se ha calculado tomando en cuenta los dos tipos de semana (ver TABLA I) y la cantidad de lámparas (TABLA IV); los resultados correspondientes a cantidad de horas de consumo por tipo de ambiente/edificio y cantidad de horas de consumo por tipo de lámparas/edificio se presentan respectivamente en las tablas VII y VIII.

TABLA V

Características fotométricas nominales de lámparas

\begin{tabular}{|c|c|c|c|c|c|c|c|}
\hline 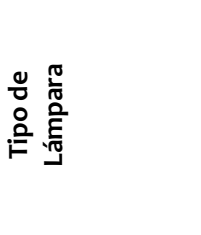 & 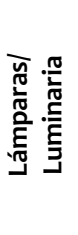 & 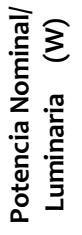 & 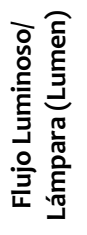 & 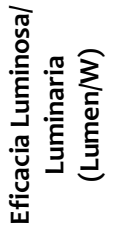 & 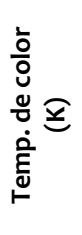 & 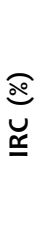 & 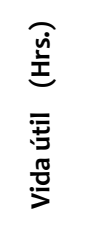 \\
\hline LED 1700 (20) & 2 & 40 & 1700 & 85.00 & 6000 & 80 & 50000 \\
\hline LED 1600 (16) & 2 & 32 & 1600 & 100.00 & 6000 & 73 & 15000 \\
\hline $\begin{array}{l}\text { LED panel } \\
\text { cuadrado }\end{array}$ & 1 & 41 & 3400 & 82.93 & 6500 & 80 & 25000 \\
\hline $\begin{array}{l}\text { LED panel } \\
\text { rectangular }\end{array}$ & 1 & 36 & 4000 & 111.11 & 4000 & 80 & 25000 \\
\hline $\begin{array}{l}\text { LED panel } \\
\text { circular }\end{array}$ & 1 & 18 & 1450 & 80.56 & 6000 & 80 & 25000 \\
\hline LED dicroič̄ & 1 & 5 & 435 & 87.00 & 6500 & 80 & 15000 \\
\hline LED reflecto & 1 & 110 & 11000 & 100.00 & 6500 & 80 & 20000 \\
\hline T12m (40W', & 2 & 100 & 2250 & 45.00 & 4100 & 89 & 20000 \\
\hline $\mathrm{T} 8 \mathrm{~m}(36 \mathrm{~W})$ & 2 & 92 & 3350 & 72.83 & 4000 & 80 & 15000 \\
\hline
\end{tabular}

\begin{tabular}{cccccccc} 
T8e $(36 \mathrm{~W})$ & 2 & 69.7 & 3350 & 96.13 & 4000 & 80 & 15000 \\
T8m60 (18W & 2 & 54 & 1350 & 50.00 & 4000 & 80 & 15000 \\
T8e60 (18W & 2 & 50 & 1350 & 54.00 & 4000 & 80 & 15000 \\
T5e120 (28W & 2 & 65.4 & 2900 & 88.69 & 4000 & 80 & 20000 \\
T5e060 (14W & 4 & 66.2 & 1350 & 81.57 & 4000 & 80 & 20000 \\
Circular (32W & 1 & 42 & 1750 & 41.67 & 6200 & 72 & 13000 \\
LFC (20W) & 1 & 20 & 1200 & 60.00 & 6500 & 80 & 8000 \\
SAP 150W & 1 & 169 & 17700 & 104.73 & 2000 & 25 & 28000 \\
MAP 250W & 1 & 286 & 5500 & 19.23 & 3400 & 63 & 10000 \\
\hline
\end{tabular}

Nota: Elaboración propia y datos de fabricantes.

TABLA VI

Potencia Eléctrica nominal y medida de lámparas

\begin{tabular}{|c|c|c|c|c|c|}
\hline $\begin{array}{l}\text { Tipo de } \\
\text { Lámpara }\end{array}$ & 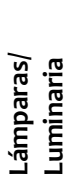 & $\begin{array}{l}\text { Potencia } \\
\text { Nominal/ } \\
\text { Luminaria } \\
\text { (W) }\end{array}$ & $\begin{array}{l}\text { Potencia } \\
\text { Medida/ } \\
\text { Luminaria } \\
\text { 1ra. hora } \\
\text { (W) }\end{array}$ & $\begin{array}{l}\text { Potencia } \\
\text { Medida/ } \\
\text { Luminaria } \\
\text { 2da. hora } \\
\text { (W) }\end{array}$ & $\begin{array}{l}\text { Potencia } \\
\text { Medida/ } \\
\text { Luminaria } \\
\text { 3ra. hora } \\
\text { (W) }\end{array}$ \\
\hline LED 1700 & 2 & 40 & 37.92 & 37.81 & 37.80 \\
\hline LED 1600 & 2 & 32 & 30.96 & 30.62 & 30.70 \\
\hline $\begin{array}{l}\text { LED panel } \\
\text { cuadrado }\end{array}$ & 1 & 41 & 38.45 & 38.23 & 38.22 \\
\hline $\begin{array}{l}\text { LED panel } \\
\text { rectangular }\end{array}$ & 1 & 36 & 36.00 & 36.00 & 36.00 \\
\hline $\begin{array}{l}\text { LED panel } \\
\text { circular }\end{array}$ & 1 & 18 & 18.00 & 18.00 & 18.00 \\
\hline LED dicroica & 1 & 5 & 5.00 & 5.00 & 5.00 \\
\hline LED reflector & 1 & 110 & 110.00 & 110.00 & 110.00 \\
\hline T12m & 2 & 100 & 100.05 & 101.18 & 101.08 \\
\hline T8m & 2 & 92 & 94.17 & 94.58 & 95.01 \\
\hline T8e & 2 & 69.7 & 67.35 & 67.23 & 67.01 \\
\hline T8m6o & 2 & 54 & 54.00 & 54.00 & 54.00 \\
\hline T8e6o & 2 & 50 & 50.00 & 50.00 & 50.00 \\
\hline T5e120 & 2 & 65.4 & 62.85 & 63.08 & 63.02 \\
\hline T5e060 & 4 & 66.2 & 66.25 & 66.57 & 66.50 \\
\hline Circular & 1 & 42 & 42.00 & 42.00 & 42.00 \\
\hline LFC & 1 & 20 & 20.00 & 20.00 & 20.00 \\
\hline SAP $150 W$ & 1 & 169 & 169.00 & 169.00 & 169.00 \\
\hline MAP $250 \mathrm{~W}$ & 1 & 286 & 286.00 & 286.00 & 286.00 \\
\hline
\end{tabular}

Nota: Elaboración propia y datos de fabricantes

TABLA VI

Cantidad de Horas de consumo por tipo de ambiente/edificio

\begin{tabular}{lrrrrrr}
\hline \multicolumn{1}{c}{ Ambientes } & Edif.1 & Edif.2 & Edif.3 & Edif.4 & Edif.5 & Total \\
\hline Aula & 488600 & 165120 & 163800 & 0 & 22960 & 840480 \\
Laboratorio & 0 & 204880 & 0 & 267760 & 0 & 472640 \\
Biblioteca & 0 & 0 & 0 & 0 & 409760 & 409760 \\
Auditorio & 84720 & 0 & 0 & 0 & 0 & 84720 \\
Sala de & 23400 & 0 & 0 & 0 & 0 & 23400 \\
reuniones & & & & 0 & & \\
Oficina & 553440 & 22000 & 0 & 44160 & 144768 & 764368 \\
Almacén & 35256 & 0 & 0 & 0 & 0 & 35256 \\
Archivo & 14040 & 0 & 0 & 0 & 12480 & 26520 \\
Cafetería & 3952 & 0 & 14560 & 0 & 0 & 18512 \\
Patio & 449176 & 182728 & 224224 & 112112 & 0 & 968240 \\
Pasadizo & 0 & 37440 & 50544 & 0 & 7488 & 95472 \\
Escalera & 26208 & 16848 & 41184 & 0 & 6240 & 90480 \\
Baño & 95760 & 10400 & 15600 & 0 & 16952 & 138712 \\
\hline & 1774552 & 639416 & 509912 & 424032 & 620648 & 3968560 \\
\end{tabular}

Nota: Elaboración propia y datos FIA.

El inventario de las emisiones indirectas de GEI por energía asociada con la generación de electricidad de una fuente externa se presentan en las tablas IX y $X$. 


\section{j. El año base histórico seleccionado y el inventario de GEI para el año base (véase [7] numeral 5.3.1)}

El año base considerado es 2018, debido a que este año se aprobó el proyecto 'Huella de carbono Emisiones de GEI por uso del Sistema de iluminación de la Facultad de Ingeniería Ambiental de la Universidad Nacional de Ingeniería, Lima-Perú', que comprende el estudio de emisiones de GEI realizado.

Las actividades se realizan en 5 edificaciones, las cuales comprenden 1509 lámparas (568 LED, 941 de descarga) y alberga 1225 usuarios (estudiantes, docentes, administrativos y de apoyo).

TABLA VIII

Cantidad de Horas de consumo por tipo de lámparas/edificio

\begin{tabular}{lcccccc}
\hline Lámparas & Edif.1 & Edif.2 & Edif.3 & Edif.4 & Edif.5 & Total \\
\hline LED 1700 & 195020 & 44000 & 0 & 0 & 0 & 239020 \\
LED 1600 & 690892 & 485744 & 22464 & 0 & 0 & 1199100 \\
LED panel & 81312 & 0 & 0 & 0 & 0 & 81312 \\
cuadrado & & & & & & \\
LED panel & 1440 & 0 & 0 & 0 & 0 & 1440 \\
rectangular & & & & & & \\
LED panel & 21060 & 0 & 10920 & 0 & 0 & 31980 \\
circular & & & & & & \\
LED dicroica & 25740 & 0 & 0 & 0 & 0 & 25740 \\
LED reflector & 4368 & 2184 & 0 & 0 & 0 & 6552 \\
T12m & 245932 & 0 & 0 & 8000 & 310832 & 564764 \\
T8m & 206268 & 5200 & 1872 & 0 & 297128 & 510468 \\
T8e & 69352 & 70432 & 52416 & 116960 & 0 & 309160 \\
T8m60 & 2320 & 0 & 0 & 0 & 0 & 2320 \\
T8e60 & 14040 & 0 & 0 & 0 & 0 & 14040 \\
T5e120 & 47040 & 0 & 418600 & 299072 & 0 & 764712 \\
T5e060 & 132640 & 0 & 0 & 0 & 0 & 132640 \\
Circular & 0 & 2320 & 0 & 0 & 12688 & 15008 \\
LFC & 30576 & 27352 & 3640 & 0 & 0 & 61568 \\
SAP 150W & 2184 & 2184 & 0 & 0 & 0 & 4368 \\
MAP 250W & 4368 & 0 & 0 & 0 & 0 & 4368 \\
\hline & 1774552 & 639416 & 509912 & 424032 & 620648 & 3968560 \\
\hline
\end{tabular}

Nota: Elaboración propia y datos FIA

La metodología de cuantificación aplicada corresponde a cálculos basados en datos de la actividad de GEI multiplicados por el factor de emisión (ver Fig. 2), obteniéndose el inventario de emisiones de GEI por ambiente de cada edificio (ver TABLA IX), las emisiones por tipo de lámpara existentes en cada edificio (ver TABLA X), así como la emisión por cada edificio y el total de los 5 edificios que corresponde al Alcance 2.

TABLA IX

Emisiones/Ambiente (tonCO2e)

\begin{tabular}{|c|c|c|c|c|c|c|}
\hline Ambientes & $\begin{array}{c}\text { Edif. } \\
1\end{array}$ & $\begin{array}{c}\text { Edif. } \\
2\end{array}$ & $\begin{array}{c}\text { Edif. } \\
3 \\
\end{array}$ & $\begin{array}{c}\text { Edif. } \\
4 \\
\end{array}$ & $\begin{array}{c}\text { Edif. } \\
5\end{array}$ & Total \\
\hline Aula & 4.646 & 1.398 & 2.688 & & 0.599 & 9.330 \\
\hline Laboratorio & & 1.640 & & 4.601 & & 6.242 \\
\hline Biblioteca & & & & & 10.438 & 10.438 \\
\hline Auditorio & 0.961 & & & & & 0.961 \\
\hline $\begin{array}{l}\text { Sala de } \\
\text { reuniones }\end{array}$ & 0.156 & & & & & 0.156 \\
\hline Oficina & 11.671 & 0.383 & & 0.725 & 3.706 & 16.486 \\
\hline Almacén & 0.877 & & 0.000 & & & 0.877 \\
\hline Archivo & 0.351 & & & & 0.319 & 0.670 \\
\hline Cafetería & 0.074 & & 0.140 & & & 0.214 \\
\hline Patio & 4.774 & 2.387 & 3.684 & 1.842 & & 12.687 \\
\hline Pasadizo & & 0.300 & 0.673 & & 0.197 & 1.170 \\
\hline Escalera & 0.210 & 0.140 & 0.717 & & 0.137 & 1.203 \\
\hline \multirow[t]{2}{*}{ Baño } & 1.983 & 0.083 & 0.256 & & 0.412 & 2.735 \\
\hline & 25.703 & 6.331 & 8.157 & 7.169 & 15.808 & 63.169 \\
\hline
\end{tabular}

Nota: Elaboración propia.

TABLAX

Emisiones / tipo de lámpara (tonCO2e)

\begin{tabular}{|c|c|c|c|c|c|c|}
\hline Lámparas & Edif.1 & Edif.2 & Edif.3 & Edif.4 & Edif.5 & Total \\
\hline LED 1700 & 1.920 & 0.434 & & & & 2.354 \\
\hline LED 1600 & 5.534 & 3.884 & 0.180 & & & 9.598 \\
\hline $\begin{array}{l}\text { LED panel } \\
\text { cuadrado }\end{array}$ & 1.621 & & & & & 1.621 \\
\hline $\begin{array}{l}\text { LED panel } \\
\text { rectangular }\end{array}$ & 0.027 & & & & & 0.027 \\
\hline $\begin{array}{l}\text { LED panel } \\
\text { circular }\end{array}$ & 0.198 & & 0.102 & & & 0.300 \\
\hline LED dicroica & 0.067 & & & & & 0.067 \\
\hline LED reflector & 0.251 & 0.125 & & & & 0.376 \\
\hline T12m & 6.475 & & & 0.211 & 8.179 & 14.864 \\
\hline $\mathrm{T} 8 \mathrm{~m}$ & 5.102 & 0.129 & 0.046 & & 7.351 & 12.629 \\
\hline T8e & 1.213 & 1.231 & 0.917 & 2.045 & & 5.406 \\
\hline T8m6o & 0.033 & & & & & 0.033 \\
\hline T8e6o & 0.183 & & & & & 0.183 \\
\hline T5e120 & 0.768 & & 6.874 & 4.913 & & 12.555 \\
\hline T5e060 & 1.149 & & & & & 1.149 \\
\hline Circular & & 0.051 & & & 0.278 & 0.329 \\
\hline LFC & 0.319 & 0.285 & 0.038 & & & 0.642 \\
\hline SAP $150 \mathrm{~W}$ & 0.192 & 0.192 & & & & 0.385 \\
\hline \multirow[t]{2}{*}{ MAP $250 \mathrm{~W}$} & 0.651 & & & & & 0.651 \\
\hline & 25.703 & 6.331 & 8.157 & 7.169 & 15.808 & 63.169 \\
\hline
\end{tabular}

Nota: Elaboración propia. 


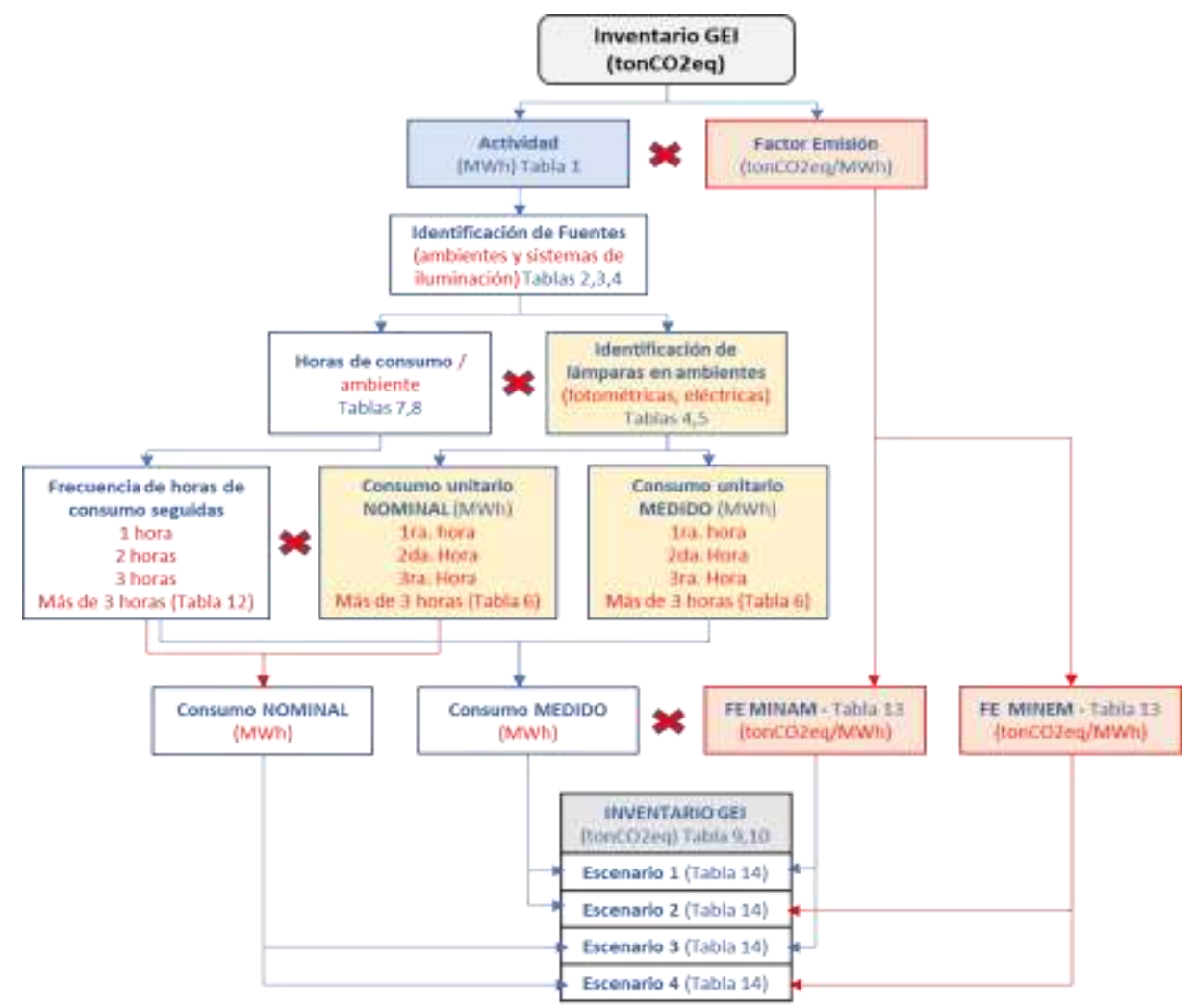

Fig. 2. Metodología de cálculo de GEl.

Finalmente, se presenta los valores de cálculo resultantes en la TABLA XI.

Total Alcance 2: TonCO2e

TABLA XI

Valores de cálculos

\begin{tabular}{|c|c|c|c|c|}
\hline Descripción & Unidades & $\begin{array}{l}\text { Semanas } \\
\text { tipo } 1\end{array}$ & $\begin{array}{l}\text { Semanas } \\
\text { tipo } 2\end{array}$ & Anual \\
\hline Cantidad Lámparas & Lámparas & 1509 & 1509 & 1509 \\
\hline $\begin{array}{l}\text { Potencia } \\
\text { Promedio/Lámpara }\end{array}$ & W & 30.256 & 30.927 & 30.526 \\
\hline $\begin{array}{l}\text { Cantidad de } \\
\text { semanas }\end{array}$ & Semanas & 28 & 24 & 52 \\
\hline $\begin{array}{l}\text { Horas Promedio } \\
\text { Uso Lámpara/Día }\end{array}$ & Horas/día & 8.026 & 6.291 & 7.225 \\
\hline $\begin{array}{l}\text { Consumo de } \\
\text { Energía }\end{array}$ & MWh (anual) & 71.821 & 49.322 & 121.143 \\
\hline Factor de Emisión & tonCO2eq/MWh & 0.52144 & 0.52144 & 0.52144 \\
\hline
\end{tabular}

\begin{tabular}{lllll}
\hline Emisión GEl & tonCO2eq & 37.451 & 25.718 & $\mathbf{6 3 . 1 6 9}$ \\
\hline
\end{tabular}

Nota: Elaboración propia.

k. Explicación de cualquier cambio en el año base o de otros datos históricos sobre los GEI y cualquier otro recálculo del año base u otro inventario histórico de GEI (véase [7] numeral 5.3.2)

No aplica por ser la presentación del año base.
I. Referencia o descripción de metodologías de cuantificación, que incluya las razones para su selección (véase [7] numeral 4.3.3)

La metodología de cuantificación aplicada es la combinación de medición y cálculo (ver Fig. 2).

Los cálculos basados en datos de las actividades de GEI multiplicados por el factor de emisión de GEI :

\section{Inventario GEI = Actividades GEI X Factor de Emisión}

Se identificaron como fuentes de emisión indirecta los sistemas de iluminación (compuestos principalmente por lámparas y equipos auxiliares) existentes en los ambientes comprendidos en los 5 edificios de la FIA (ver TABLAS III y IV) en los cuales se desarrollan actividades académicas, de gestión y apoyo (ver TABLA II), así como los de uso común (escaleras, patios, pasadizos, baños). De las fuentes identificadas se cuantificó por ambiente las horas de ocupación (según la programación de clases y horarios de trabajo), de acuerdo con esta ocupación se realizaron las mediciones de consumo intermitente de energía eléctrica por los sistemas de iluminación y el factor de emisión de GEI utilizado corresponde a la red eléctrica peruana recomendado por el Ministerio del Ambiente (MINAM). Según la programación de ocupación de aulas, éstas se usan por períodos intermitentes en el día de 1 hora a 14 horas, con 
desocupación de por lo menos 1 hora entre períodos (ver TABLA XII y Fig. 3).

TABLA XII

Frecuencia de uso semanal de ambientes

\begin{tabular}{|c|c|}
\hline $\begin{array}{l}\text { Horas de uso } \\
\text { continuo de } \\
\text { ambientes }\end{array}$ & $\begin{array}{c}\text { Ambientes de } \\
\text { mayor uso }\end{array}$ \\
\hline 1 & 3 Aulas \\
\hline 2 & 105 Aulas, almacenes \\
\hline 3 & 100.5 Aulas, auditorio \\
\hline 4 & 50 Aulas \\
\hline 5 & 35 Aulas \\
\hline 6 & 279 Aulas, áreas comunes \\
\hline 7 & 15 Aulas \\
\hline 8 & 33 Aulas, oficinas \\
\hline 9 & 152.5 Oficinas \\
\hline 10 & 390 Aulas, laboratorio, biblioteca, oficinas \\
\hline 11 & 9.5 Áreas comunes \\
\hline 12 & 53 Áulas, biblioteca, oficinas \\
\hline 13 & 7 Aulas. laboratorio \\
\hline 14 & 214 Laboratorio, áreas comunes \\
\hline
\end{tabular}

Nota: Elaboración propia y datos FIA.

Para la determinación del consumo de energía eléctrica se realizaron las mediciones a las muestras por cada tipo de lámpara siguientes: Tubulares LED1700, Tubulares LED1600, LED panel cuadrado, LED panel rectangular, Fluorescente T12m (tubular de 1.20 $\mathrm{m}$, con balasto electromagnético), Fluorescente T8m (tubular de $1.20 \mathrm{~m}$, con balasto electromagnético), Fluorescente T8e (tubular de $1.20 \mathrm{~m}$, con balasto electrónico), Fluorescente T5e120 (tubular de $1.20 \mathrm{~m} \mathrm{y}$ balasto electrónico) y Fluorescente T5e060 (tubular de $0.60 \mathrm{~m} \mathrm{y}$ balasto electrónico), por ser las más numerosas; por 3 horas seguidas, con toma de datos cada segundo, calculándose los consumos promedio para la primera hora de uso, así como para la segunda y tercera. Dadas las variaciones reales de consumo entre tipos de lámparas y durante las horas de uso (las más representativas se grafican en las Fig. 4, 5, 6, 7, 8 y 9, se ha considerado diferenciadamente estos promedios cuando el uso es por 1 hora (promedio de primera hora), por 2 horas (promedio de primera hora + promedio de segunda hora), por 3 horas (promedio de primera hora + promedio de segunda hora + promedio de tercera hora) y para más de 3 horas (promedio de primera hora + promedio de segunda hora + promedio de tercera hora + horas adicionales igual al promedio de la tercera hora), los promedios de las mediciones realizadas se presentan en la TABLA VI. Para los otros tipos de lámparas: LED panel circular, LED dicroica, LED reflector, Fluorescente T8e60 (tubular de $0.60 \mathrm{~m}$, con balasto electrónico), Fluorescente circular, Lámpara Fluorescente Compacta (LFC), Sodio de Alta Presión (SAP150W) y Mercurio de Alta Presión MAP250W); se ha considerado la potencia nominal que se detalla en la TABLA VI).

Para determinar la ocupación de ambientes durante las 52 semanas del año, se diferencia 2 tipos de ocupación semanal (ver TABLA I):
Ocupación durante las 'Semanas tipo 1': Promedio de ocupación de las 14 semanas durante clases del ciclo 2018-1 y 14 semana durante clases del ciclo 2018-2.

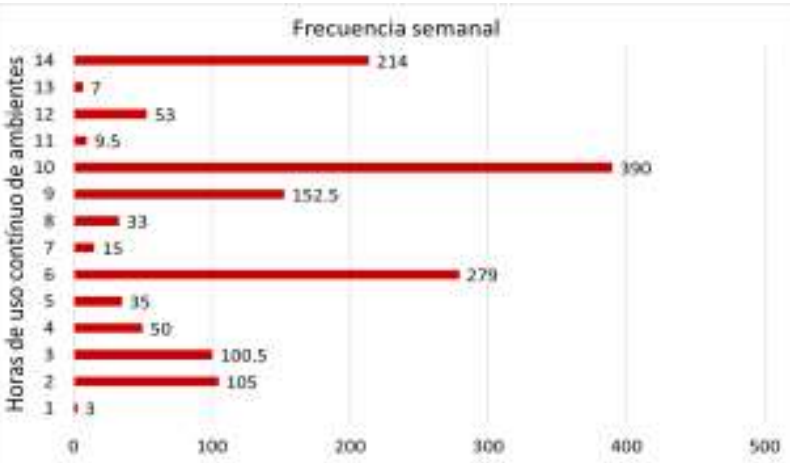

Fig. 3. Frecuencia semanal de horas de uso continuas de ambientes.

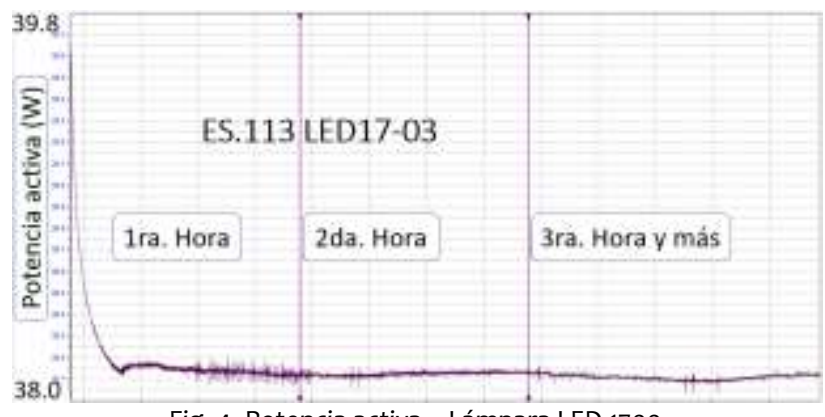

Fig. 4. Potencia activa - Lámpara LED 1700.

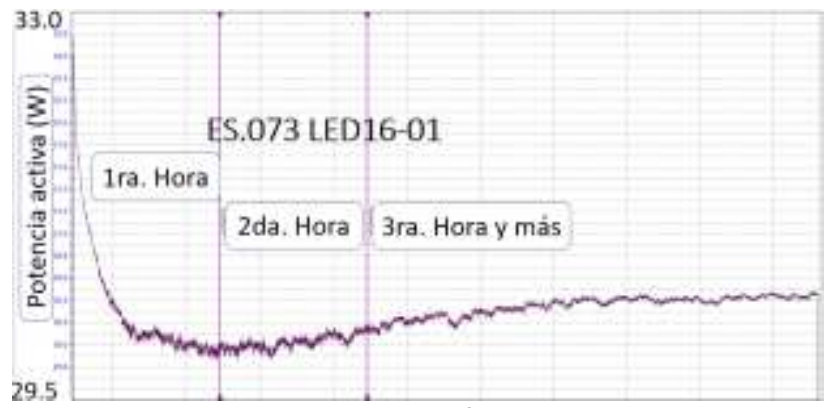

Fig. 5. Potencia activa - Lámpara LED 1600.

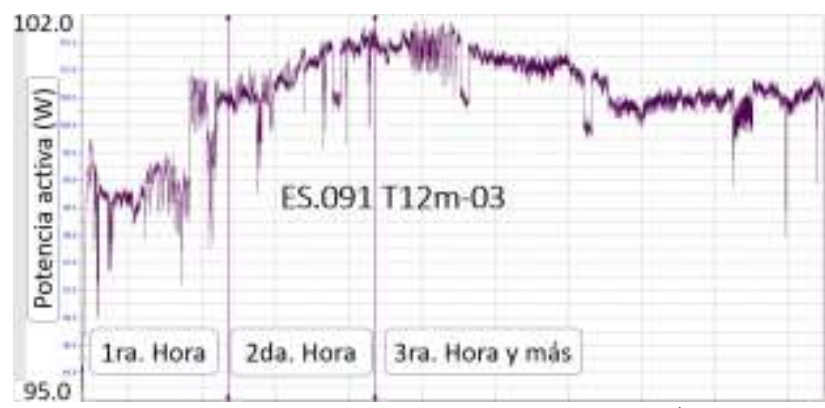

Fig 6. Potencia activa - Lámpara fluorescente T12m (balasto electromecánico) 


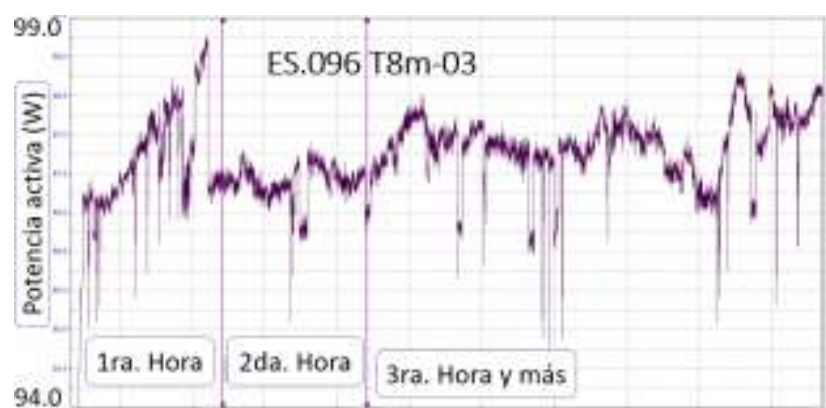

Fig. 7. Potencia activa - Lámpara fluorescente T8m (balasto electromecánico)

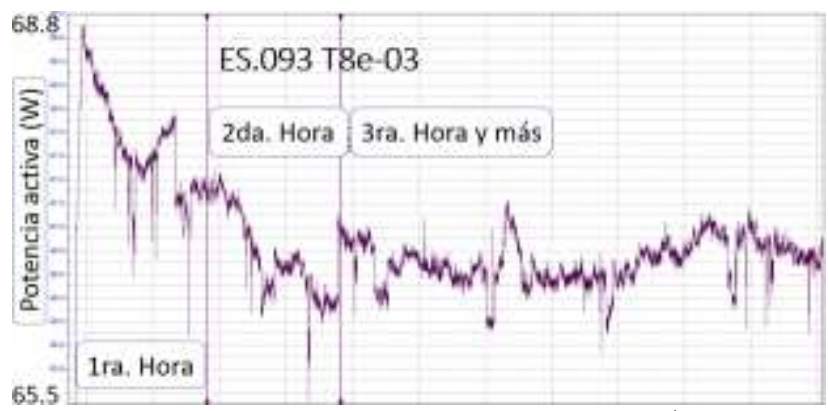

Fig. 8. Potencia activa - Lámpara fluorescente T8e (balasto electrónico).

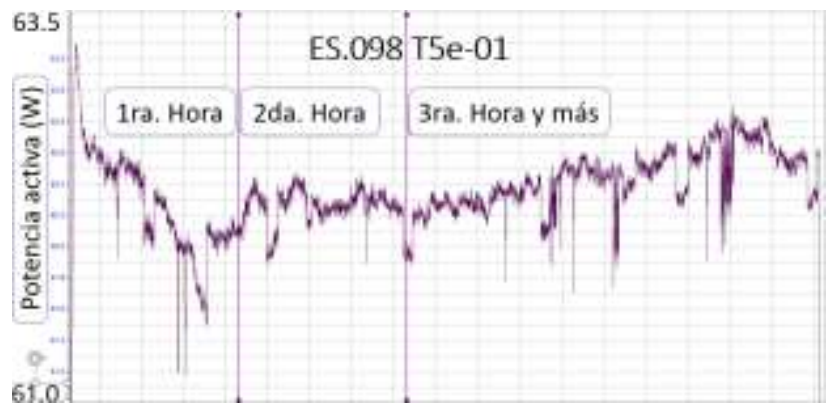

Fig. 9. Potencia activa - Lámpara fluorescente T5e (balasto electrónico).

Ocupación durante las 'Semanas tipo 2': 24 semanas restantes (durante las cuales las aulas y laboratorios, se ocupan al 50\% de lo programado para la semana tipo 1 y los otros ambientes siempre al 100\%).

La metodología de cuantificación aplicada corresponde a cálculos basados en datos de la actividad de GEI multiplicados por el factor de emisión (ver Figura 2.), obteniéndose el inventario de emisiones de GEI por ambiente de cada edificio (ver TABLA IX), las emisiones por tipo de lámpara existentes en cada edificio (ver TABLA X), así como la emisión por cada edificio y el total de los 5 edificios que corresponde al Alcance 2.

m. Explicación de cualquier cambio en la
metodología de cuantificación utilizadas
previamente (véase [7] numeral 4.3.3)

No aplica por ser la presentación del año base.

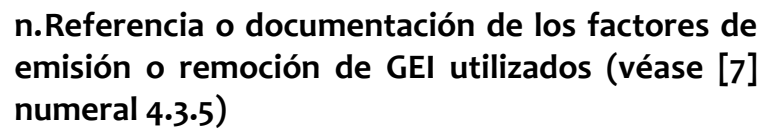

n.Referencia o documentación de los factores de numeral 4.3.5)
De acuerdo a lo informado por el Ministerio del Ambiente (MINAM), el valor del Factor de Emisión es: $\mathbf{0 . 5 2 1 4 4}$ tCO2eq/MWh [22]; es el valor más actualizado del Factor de Emisión de la red eléctrica peruana y recomendado por el MINAM; corresponde al calculado por el desarrollador del proyecto 'Central Hidroeléctrica Poechos II', para la validación MDL (Mecanismo de Desarrollo Limpio) del mismo proyecto, correspondiente al año 2017, el cual es el más actualizado; este Factor de Emisión es el utilizado en los cálculos y presentados en las tablas IX, X, XI, XIII y $\mathrm{XIV}$, y en los cálculos de los Escenarios 1 y 2.

\section{o.Descripción del impacto de las incertidumbres en la exactitud de los datos de emisiones y remociones de GEI (véase [7] numeral 5.4)}

De acuerdo a lo informado por el Ministerio del Ambiente (MINAM), el valor del Factor de Emisión es referencial para su uso en proyectos debido a que no existe un valor oficial para el país, así mismo, que existen numerosas fuentes nacionales e internacionales que pueden ser utilizadas como referencia para nuestro propósito [22].

El valor más actualizado del Factor de Emisión de la red eléctrica peruana y recomendado por el MINAM es el calculado por el desarrollador del proyecto 'Central Hidroeléctrica Poechos II', para la validación MDL (Mecanismo de Desarrollo Limpio) del mismo proyecto, correspondiente al año 2017, el cual es el más actualizado, cuyo valor es: $\mathbf{0 . 5 2 1 4 4}$ tCO2eq/MWh [22].; este Factor de Emisión es el utilizado en los cálculos y presentados en las tablas IX, X, XI, XIII y XIV, y en los cálculos de los Escenarios 1 y 2.

También existe el Factor de Emisión de la energía eléctrica de la red, calculado por el Ministerio de Energía y Minas (MINEM) para el año 2016, cuyo valor es: 0.4367 tCO2e/MWh [22]; este Factor de Emisión es el utilizado en los cálculos y presentados en las tablas XIII, XIV y en los cálculos de los Escenarios 3 y 4 [22]; así mismo, este factor de emisión es calculado por los proponentes de los proyectos de Mecanismo de Desarrollo Limpio (MDL).

El impacto de esta incertidumbre se presenta en los siguientes escenarios, así como los resultados que muestran las diferencias (ver TABLAS XIII y XIV):

\section{TABLA XIII}

Escenarios (tCO2eq) según F.E. y Potencia de lámparas y equipos auxiliares

\begin{tabular}{ccc}
\hline Factor de Emisión & $\begin{array}{c}\text { Potencia } \\
\text { Medida }\end{array}$ & $\begin{array}{c}\text { Potencia } \\
\text { Nominal }\end{array}$ \\
\hline $\begin{array}{c}\text { F.E. MINAM: } 0.52144 \\
\text { tCO2eq/MWh }\end{array}$ & Escenario 1 & Escenario 2 \\
$\begin{array}{c}\text { F.E. MINEM: } 0.4367 \\
\text { tCO2eq/MWh }\end{array}$ & Escenario 3 & Escenario 4 \\
\hline
\end{tabular}


Nota: Elaboración propia.

De las tablas XIII y XIV, tomando como referencia el escenario 1 (F.E. MINAM y potencia medida) la mayor diferencia se da con el escenario 3 (F.E. MINEM y potencia medida) $16.25 \%$, siendo de mayor impacto la diferencia de valores de los Factores de Emisión, comprobamos la necesidad de tener un único Factor de Emisión de GEI del Sistema Interconectado Nacional, por lo que se debería insistir al Ministerio del Ambiente (MINAM) su determinación; esto permitiría homologar el cálculo de inventarios de diferentes organizaciones y tener información apropiada para la toma de decisiones sobre la gestión de la huella de carbono. $Y$ respecto al escenario 2 (F.E MINAM y potencia nominal) la diferencia es $1.28 \%$, esto debido a que ambos escenarios se ha considerado la potencia de la lámpara y de los equipos auxiliares, por lo que la importancia de realizar mediciones directas es su contribución para dar un valor más cercano al real.

TABLA XIV

Emisiones de GEI en TonCOzeq - Según Escenarios

\begin{tabular}{lccccccc}
\hline Escenario & Edif.1 & Edif.2 & Edif.3 & Edif.4 & Edif.5 & Total & $\%$ \\
\hline Escenario 1 & 25.703 & 6.331 & 8.157 & 7.169 & 15.808 & 63.169 & 100.0 \\
Escenario 2 & 26.015 & 6.563 & 8.460 & 7.433 & 15.504 & 63.976 & 101.28 \\
Escenario 3 & 21.526 & 5.302 & 6.832 & 6.004 & 13.239 & 52.903 & 83.75 \\
Escenario 4 & 21.787 & 5.496 & 7.085 & 6.225 & 12.985 & 53.579 & 84.82 \\
\hline Nota: Elaboración propia. & & & & &
\end{tabular}

p.Una declaración de que al informe sobre GEI se ha preparado de acuerdo con esta parte de la Norme ISO 14064

Este informe ha sido desarrollado de acuerdo con los principios y requerimientos para la presentación de informes sobre los inventarios de Gases Efecto Invernadero establecidos en la Norma ISO 14064-1:2006 ('Gases de efecto invernadero - Parte 1: Especificación con orientación, a nivel de las organizaciones, para la cuantificación y el informe de las emisiones y remociones de gases de efecto invernadero').

q.Una declaración que describa si el inventario de GEI, el informe o la declaración de GEI se ha verificado, incluyendo el tipo de verificación y el nivel de aseguramiento logrado

Este informe no se ha verificado ya que es un inventario parcial, el cual debería ser complementado con futuros proyectos y luego de completado estaría en condiciones de ser verificado. Este informe corresponde al aporte parcial a la Huella de Carbono por otras emisiones indirectas de GEI que provienen de la generación de electricidad consumida por el Sistema de lluminación, en el desarrollo de las actividades académicas, de gestión y apoyo al interior de los ambientes de la Facultad de ingeniería Ambiental (FIA).

\section{MEJORAS}

El determinar el inventario de emisiones de GEI por el uso de los Sistemas de Iluminación en los ambientes de las edificaciones de la Facultad de Ingeniería Ambiental (FIA) de la Universidad Nacional de Ingeniería (UNI), en el desarrollo de las actividades académicas, gestión y de apoyo contribuye parcialmente a establecer la Línea Base de la Huella de Carbono. El estudio ha permitido identificar y estimar las emisiones indirectas de GEI que provienen de la generación de electricidad de origen externo (Alcance 2.) lo cual permite analizar la forma de consumo de los sistemas de iluminación y tener una trazabilidad de las actividades que permite seguir planteando alternativas de disminución de las emisiones indirectas de GEI en las fuentes más significativas.

\subsection{INDICADORES}

Para este propósito se ha determinado los siguientes indicadores (ver TABLAS XV y XVI) que reflejan el estado actual, los cuales debemos comparar con el estado luego de implementar las propuestas de acciones de disminución, así como de cada año:

TABLA XV

Datos e indicadores FIA 2018

\begin{tabular}{lrc}
\hline \multicolumn{1}{c}{ Indicador } & FIA & UNI \\
\hline Estudiantes (Pregrado + Posgrado) & 1036 & \\
Docentes (Pregrado + Posgrado) & 141 & \\
Trabajadores & 47 & \\
Usuarios (Estudiantes+Docentes+Trabajadores) & 1224 & \\
Lámparas (LED + Descarga) & 1509 & 29264 \\
Lámparas LED & 568 & 3571 \\
Facultades (1 de 11) & $1(9.09 \%)$ & $11(100 \%)$ \\
Carreras (3 de 28) & $3(10.71 \%)$ & $28(100 \%)$ \\
GEI total (tonCO2e) & 63.169 & \\
Promedio GEl/Estudiante (tonCO2e) & 0.061 & \\
Promedio GEI/Usuario (tonCO2e) & 0.052 & \\
Promedio GEI/Lámpara (tonCO2e) & 0.042 & \\
Lámparas FIA/ Lámparas UNI & $5.16 \%$ & \\
Lámparas LED FIA/ Lámparas FIA & $37.64 \%$ & \\
Lámparas LED FIA/ Lámparas LED UNI & $15.91 \%$ & \\
Promedio Lámparas FIA/ Usuario & 1.233 & \\
\hline NOta ElabOracion propia. &
\end{tabular}

Nota: Elaboración propia. 
TABLA XVI

Emisiones / tipo de lámpara (tonCO2e)

\begin{tabular}{|c|c|c|c|c|c|c|}
\hline Lámparas & $\begin{array}{c}\mathrm{Wh} / \\
\text { luminaria }\end{array}$ & $\begin{array}{c}10^{-06} \text { tCO2eq/ } \\
\text { Luminaria }\end{array}$ & $\begin{array}{c}\text { Flujo Luminoso/ } \\
\text { Lámpara (lúmenes) }\end{array}$ & $\begin{array}{l}10^{-09} \mathrm{tCO} e q / \\
\text { lumen }\end{array}$ & $\begin{array}{c}\text { Eficacia Luminosa } \\
\text { NOMINAL MEDIDA/ } \\
\text { Luminaria } \\
\text { (Lumen/W) }\end{array}$ & $\begin{array}{l}\text { Disminución de } \\
\text { emisiones al } \\
\text { reemplazar por } \\
\text { LED1600 }\end{array}$ \\
\hline LED 2500 & 32 & 16.686 & 2500 & 003.337 & 156.25 & \\
\hline LED 2100 & 36 & 18.772 & 2100 & 004.469 & 116.67 & \\
\hline LED 1700 & 37.92 & 19.773 & 1700 & 005.816 & 89.66 & $13 \%$ \\
\hline LED 1600 & 30.96 & 16.144 & 1600 & 005.045 & 103.36 & $0 \%$ \\
\hline LED panel cuadrado & 38.45 & 20.049 & 3400 & 005.897 & 88.43 & $14 \%$ \\
\hline LED panel rectangular & 36.00 & 18.772 & 4000 & 004.693 & 111.11 & $-7 \%$ \\
\hline $\mathrm{T} 12 \mathrm{~m}$ & 100.05 & 52.170 & 2250 & 011.593 & 44.98 & $56 \%$ \\
\hline $\mathrm{T} 8 \mathrm{~m}$ & 94.17 & 49.104 & 3350 & 007.329 & 71.15 & $31 \%$ \\
\hline T8e & 67.35 & 35.119 & 3350 & 005.242 & 99.48 & $4 \%$ \\
\hline T5e120 & 62.85 & 32.773 & 2900 & 005.650 & 92.28 & $11 \%$ \\
\hline
\end{tabular}

Nota: Elaboración propia.

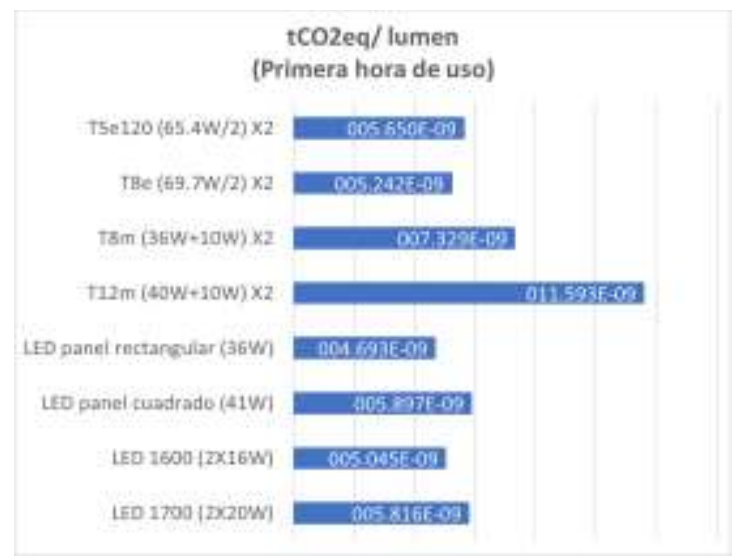

Fig. 10. Emisiones de GEI/lumen por tipo de lámpara

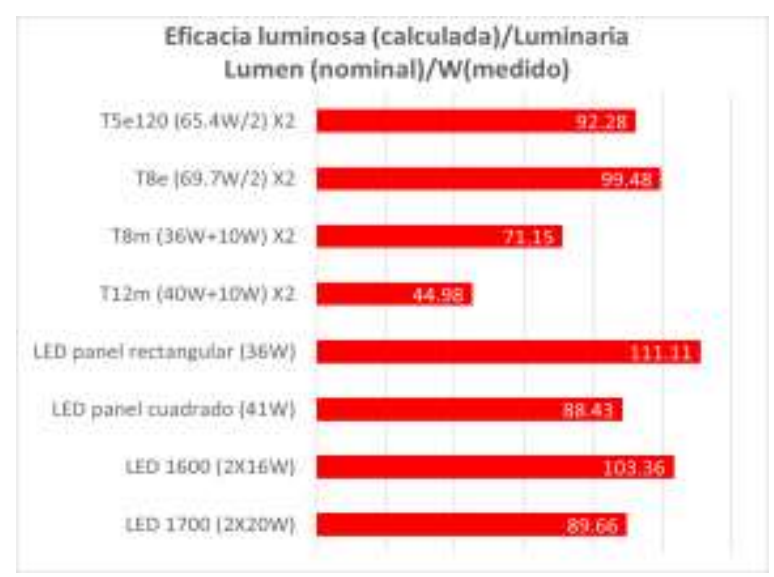

Fig. 11. Eficacia luminosa por tipo de lámpara.

Vistos los indicadores, existe la oportunidad de implementar mejoras tanto tecnológicas como de gestión de uso:

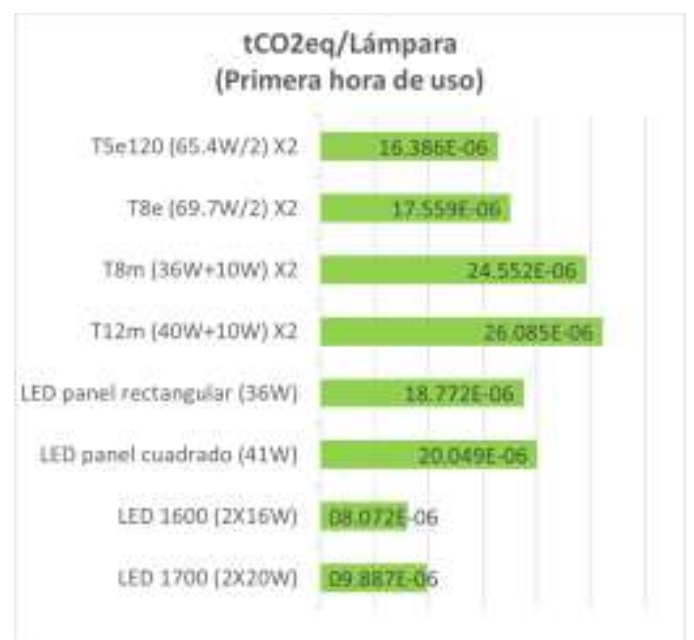

Fig. 12. Emisiones de GEl por tipo de lámpara.

\subsection{MEJORAS - TECNOLOGÍA EFICIENTE}

- Prioritariamente, en la medida que se vaya cumpliendo la vida útil, debe reemplazarse las lámparas Fluorescentes T12 y los balastos electromagnéticos, así como adquirir equipos con etiqueta de eficiencia energética para reducir las emisiones de GEI, en cumplimiento del Decreto Supremo No 034-08-EM 'Medidas para el ahorro de energía en el sector público’ [23].

- Es necesario continuar el reemplazo de lámparas por tecnología LED, considerando la siguiente prioridad: Reemplazo al corto plazo de 219 lámparas fluorescentes T12 y 173 lámparas fluorescentes T8 con balasto electromagnético, existentes en aulas, bibliotecas y oficinas; reemplazo progresivo de 118 lámparas fluorescentes T8 con balasto electrónico existentes en laboratorios y oficinas.

- Reemplazo de lámparas de sodio y mercurio por reflectores LED. y las que se vaya cumpliendo su vida útil por lámparas LED. 
- Reemplazo en el mediano plazo de 431 lámparas de descarga por lámparas LED, existentes en aulas, laboratorios, auditorio, oficinas y áreas comunes, en la medida que se vaya cumpliendo la vida útil de los equipos de iluminación actualmente en uso (ver TABLA IV).

- En cuanto a las lámparas existentes en la FIA, la que unitariamente genera la menor emisión indirecta de GEI (ver Fig. 10) es la de mayor eficacia luminosa (ver Fig. 11 y 12), ya que para los mismos niveles de iluminación requieren menor potencia por unidad de tiempo, para el año base 2018 es la lámpara tipo LED1600, de 100 lúmenes/W (ver TABLA XVI). No obstante, ya existen comercialmente lámparas LED de mayores eficacias que deberían tomarse en cuenta para futuros reemplazos.

- Cada reemplazo por lámparas LED16 representa unitariamente (ver TABLA XVI):

- El reemplazo de lámparas fluorescentes T12 con balasto electromagnético representa una disminución de $56 \%$ de emisiones.

- El reemplazo de lámparas fluorescentes T8 con balasto electromagnético representa una disminución de 31\% de emisiones.

- El reemplazo de lámparas fluorescentes T8 con balasto electrónico representa una disminución de $4 \%$ de emisiones.

- El reemplazo de lámparas fluorescentes T5 con balasto electrónico representa una disminución de $11 \%$ de emisiones.

- Incentivar proyectos para realizar el inventario de GEI en forma continuada los siguientes años, para lo cual se debería realizar con un Factor de Emisión de GEI del Sistema Interconectado Nacional único, por lo que se debería insistir al Ministerio del Ambiente (MINAM) su determinación. Esto permitiría homologar el cálculo de inventarios de diferentes organizaciones y tener información apropiada para la toma de decisiones sobre la gestión de la huella de carbono.

- Se requiere un estudio de niveles de iluminancia, que comprenda la medición y diagnóstico a fin de que las mejoras que se propongan consideren los niveles de iluminación apropiados.

- Continuar con la línea de investigación de tecnologías y gestión de uso eficientes, considerando los sistemas de iluminación de estado sólido que posibilitan la 'gestión inteligente' (sensores, control y actuadores).

\subsection{MEJORA - GESTIÓN DE USO EFICIENTE:}

- Las universidades y en especial las facultades relacionadas a la Ingeniería Ambiental tienen la responsabilidad relevante de contribuir a la sostenibilidad ambiental a través de la formación académica de profesionales, con investigaciones y acciones directas al respecto; las cuales servirán académicamente para replicar las buenas prácticas en otras facultades, universidades y sectores. Esta contribución está alineada con las acciones de mitigación conformantes de las 'Contribuciones Nacionalmente Determinadas' (NDC), que constituyen el compromiso del Perú para reducir sus emisiones de gases de efecto invernadero al año 2030, en el marco del Acuerdo de París.

- Establecer políticas de programación de ocupación y uso de iluminación de ambientes.

- Desarrollar campañas de capacitación en el uso eficiente de los sistemas de iluminación y concientización de los beneficios.

\section{CONCLUSIONES}

Este proyecto presenta el estudio parcial de la huella de carbono, correspondiente a las emisiones indirectas de GEI por uso del sistema de iluminación de la Facultad de Ingeniería Ambiental de la Universidad Nacional de Ingeniería, ubicada en el campo universitario en la ciudad de Lima-Perú.

- Para nuestro estudio aplicamos la norma ISO 140641, debido a que comprende la identificación y cuantificación de fuentes y el informe de los inventarios de GEI correspondientes parcialmente a las actividades de la organización, específicamente por el uso de los sistemas de iluminación de la Facultad de Ingeniería Ambiental de la Universidad Nacional de Ingeniería.

- Se identifica como fuente de emisión indirecta de GEI al aporte a la Huella de Carbono por emisiones indirectas de GEI que provienen de una fuente externa de generación de electricidad consumida por las lámparas del Sistema de Iluminación, al interior de los ambientes de la Facultad de Ingeniería Ambiental (FIA).

- La metodología de cuantificación aplicada es la combinación de medición y cálculo (ver Fig. 2). Los cálculos basados en datos de las actividades de GEI, datos de las horas de consumo de energía eléctrica y las mediciones intermitentes de consumo de energía por los sistemas de iluminación, multiplicados por los factores de emisión de GEI.

- El valor más actualizado del Factor de Emisión de la red eléctrica peruana y recomendado por el MINAM es el calculado por el desarrollador del proyecto 'Central Hidroeléctrica Poechos II', para la validación MDL (Mecanismo de Desarrollo Limpio) del mismo proyecto, correspondiente al año 2017, el cual es el más actualizado, cuyo valor es: 0.52144 tCO2eq/MWh [22].

- Se estableció la Línea Base de la Huella de Carbono por el uso de los Sistemas de Iluminación en los ambientes de las edificaciones de la Facultad de Ingeniería Ambiental - UNI, en el desarrollo de sus actividades académicas, gestión y de apoyo; fijándose el 2018 como el año base, 
determinándose el inventario de emisiones de GEI por uso del Sistema de iluminación de la Facultad de Ingeniería Ambiental de la Universidad Nacional de Ingeniería, en las actividades académicas, de gestión y apoyo, de 63.169 tonCOze.

- La mayor emisión es debido al desarrollo de la actividad académica directa realizada en aulas, laboratorios y bibliotecas (41.18\%), seguida por la actividad en oficinas (26.10\%) y accesos a ambientes comunes: patios, pasadizos y escaleras (23.84\%).

- Se tiene una emisión per cápita de 0.061 tonCO2e/estudiante y 0.052 tonCO2e/usuario, así como un promedio de 0.042 tonCO2e/lámpara.

- Las actividades se realizan en 5 edificaciones las cuales comprenden 1509 lámparas (568 LED, 941 de descarga) y alberga 1225 usuarios (estudiantes, docentes, administrativos y de apoyo).

- Con relación a las 11 facultades de la universidad la facultad representa $9.09 \%$, el $10.71 \%$ de las 28 carreras, $7.23 \%$ de 11777 estudiantes. Sin embargo, la FIA tiene $5.16 \%$ de 29264 lámparas (por debajo del promedio), 15.91\% de 3571 lámparas LED (por encima del promedio), lo cual muestra que es una de las facultades con mayor avance en el reemplazo de lámparas por tecnología más eficiente y de menores emisiones indirectas en el consumo de energía eléctrica generada exteriormente.

- En cuanto a las lámparas existentes en la FIA, la que unitariamente genera la menor emisión indirecta de GEI es la de mayor eficacia luminosa, ya que para para los mismos niveles de iluminación requieren menor potencia por unidad de tiempo, para este año base es la tipo LED1600 de 100 lúmenes/W. No obstante, ya existen comercialmente lámparas LED de mayores eficacias que deberían tomarse en cuenta para futuros reemplazos.

- No obstante, la lámpara de menor emisión y mayor eficacia es la tipo LED panel rectangular no se ha tomado como referencia ya existe solo una y es del tipo empotrable en falso techo, que no es aplicable a las características de la mayoría de los ambientes.

- El reemplazo de lámpara LED por Fluorescente T12 representa una disminución unitaria de 56\% de emisiones, por Fluorescente T8 de 31\%.

- Existen 219 lámparas fluorescentes T12 y 173 lámparas fluorescentes T8 con balasto electromagnético, que prioritariamente deben reemplazarse, para reducir las emisiones en cumplimiento del Decreto Supremo $N^{\circ}$ 034-08-EM [23].

- Se identificaron oportunidades de mejora, tanto para incrementar el uso de tecnología eficiente y gestión de uso eficiente. Este estudio pretende servir académicamente para replicar las buenas prácticas en otras Facultades, Universidades y sectores. El desafío es aumentar en la demanda, que los sistemas utilicen la energía eléctrica de manera eficiente, en nuestro caso los sistemas de iluminación, para que nuestros sistemas energético, ambiental y económico sean sostenibles.

\section{AGRADECIMIENTOS}

Un reconocimiento especial a la Facultad de Ingeniería Ambiental por las facilidades ofrecidas para la realización del estudio, a la estudiante investigadora de la FIA UNI Karen Centurión por su apoyo en la búsqueda de información e investigación de mercado; así mismo, al técnico de la FIA UNI Víctor Diaz por su apoyo para obtener las muestras para la realización de las mediciones de consumo de energía.

\section{REFERENCIAS}

[1] Convención Marco sobre el Cambio Climático 2015, Acuerdo de París Conferencia de las Partes 21, Paris, Francia: 2015.

[2] IEEE. (2019, Nov 24). IEEE 2019 Energy Efficiency and Sustainability in Electrical Systems International Conference on Industrial Technology. Available: https://www.itba.edu.ar/intranet/icit2020/

[3] E. Saavedra, F. Rey y J. Luyo, "Sistemas de Iluminación, situación actual y perspectivas", TECNIA, vol. 26, no. 2, pp.44 62, dic. 2016.

[4] M. Rondón. (2017, Nov 14). Huella de Carbono Ministerio del Ambiente $2015 . \quad$ Available: http://www.minam.gob.pe/semanaclimatica/wpcontent/uploads/sites/104/2015/06/1La-Huella-de-Carbono-yNeutralizaci\%C3\%B3n-como-instrumentos-de-sostenibilidad.pdf

[5] Fondo Nacional del Ambiente FONAM, "Caracterización y oportunidades de ahorro de energía en las pyme”, Proyecto de Eficiencia Energética FONAM-BID/FOMIN, 2008.

[6] Ministerio de Energía y Minas MINAM. (2018, Nov 16) Transformación del mercado de iluminación en el Perú 2018. Available: http://iluminacioneficiente.minem.gob.pe//espe/noticia/mem-elabora-guia-de-iluminacion-eficiente

[7] ISO, Norma ISO 14064-1:2006 Gases de Efecto Invernadero Parte 1, 2006.

[8] P. Iturregui y J. Perla, Nuevas reglas de juego, oportunidades de negocio en el Perú. Lima, Perú: Libélula, 2016.

[9] R. Lamas, R. Vásquez y A. Bustamante. (2019, Dic 05). Introducción a la huella de carbono 2016. La ruta del clima asociación. Available: https://www.youtube.com/watch?v=29yuKti4rc\&list=PLAPOqvcj7SzGU4VhRYhZaBX7kIDW_aWCy\&i ndex $=50$

[10] Gobierno Vasco, Guía metodológica para la aplicación de la norma UNE-ISO 14064-1:2006, Bilbao, España: IHOBE, 2012.

[11] J. Magro. (2019, Nov 26). Huella de Carbono motivaciones para su cálculo, gestión y verificación AENOR 2014. Available: http://aenormas.aenor.es/es/mas-

valor/todoslosvideos/motivaciones-para-el-calculo-gestion-yverificacion-de-la-huella-de-carbono

[12] AENOR, UNE-EN ISO 14064-2:2006 Gases de Efecto Invernadero Parte 2, 2015

[13] Grupo Intergubernamental de Expertos Sobre el Cambio Climático IPCC, Cambio climático 2014 Informe de síntesis, Ginebra, Suiza: Organización Meteorológica Mundial y Programa de las Naciones Unidas para el Medio Ambiente, 2015.

[14] ICONTEC INTERNACIONAL, Curso Inventario de emisiones de Gases Efecto Invernadero a partir de la Norma ISO 14064, Lima, Perú: FONAM, 2019.

[15] ISO. (2019, Nov 29). ISO 14064-1:2018(es) Gases de Efecto Invernadero - Parte 1. Available: https://www.iso.org/standard/66453.html

[16] ISO. (2019, Nov 29). ISO 14064-2:2019 Gases de Efecto Invernadero - Parte 2. Available: https://www.iso.org/standard/66454.html

TECNIA Vol.30 N¹ Enero-Junio 2020 
[17] ISO. (2019, Nov 29). ISO 14067:2018 Gases de efecto invernadero - Huella de carbono de productos. Available: https://www.iso.org/standard/71206.html

[18] ISO. (2019, Nov 29). ISO 14064-3:2019 Gases de Efecto Invernadero - Parte 3. Available:

https://www.iso.org/standard/66455.html

[19] Oficina Central de Planificación y Presupuesto UNI, Plan Estratégico Institucional PEI UNI 2020-2023, Lima, Perú: Universidad Nacional de Ingeniería, 2019.

[20] Asamblea Estatutaria 2014, Estatuto de la Universidad Nacional de Ingeniería 2014, Lima, Perú: Universidad Nacional de Ingeniería, 2014.

[21] Congreso de la República del Perú, Ley Universitaria $N^{\circ} 30220$ 2014, Lima, Perú: El Peruano, 2014.

[22] Ministerio del Ambiente 2019. (2019, May 20). Factor de Emisión del Sistema Eléctrico Interconectado Nacional SEIN. Available: www.minam.gob.pe 20 May 2019

[23] Ministerio de Energía y Minas MINEM, Medidas para el ahorro de energía en el sector público Decreto Supremo № 034-08-EM, Lima, Perú: El Peruano, 2008.

\section{(c) (1)}

Los artículos publicados por TECNIA pueden ser compartidos a través de la licencia Creative Commons: CC BY 4.0. Permisos lejos de este alcance pueden ser consultados a través del correo revistas@uni.edu.pe 